\title{
The Localization of Long-Distance Dependency Components: Integrating the Focal-lesion and Neuroimaging Record
}

\author{
Maria M. Piñango ${ }^{1,2 *}$, Emily Finn ${ }^{1,2}$, Cheryl Lacadie ${ }^{2}$ and R. Todd Constable ${ }^{2}$ \\ ${ }^{1}$ Language and Brain Lab, Department of Linguistics, Yale University, New Haven, CT, USA, ${ }^{2}$ Interdepartmental \\ Neuroscience Program, Magnetic Resonance Research Center, Yale University, New Haven, CT, USA
}

OPEN ACCESS

Edited by:

Colin Phillips,

University of Maryland, USA

Reviewed by:

William Matchin,

University of Maryland, USA

Jonathan Brennan,

University of Michigan, USA

*Correspondence:

Maria M. Piñango

maria.pinango@yale.edu

Specialty section: This article was submitted to

Language Sciences,

a section of the journal

Frontiers in Psychology

Received: 02 July 2016 Accepted: 07 September 2016 Published: 30 September 2016

Citation:

Piñango MM, Finn E, Lacadie C and Constable RT (2016) The Localization of Long-Distance Dependency Components: Integrating the Focal-lesion and Neuroimaging Record. Front. Psychol. 7:1434 doi: 10.3389/fpsyg.2016.01434
In the sentence "The captain who the sailor greeted is tall," the connection between the relative pronoun and the object position of greeted represents a long-distance dependency (LDD), necessary for the interpretation of "the captain" as the individual being greeted. Whereas the lesion-based record shows preferential involvement of only the left inferior frontal (LIF) cortex, associated with Broca's aphasia, during real-time comprehension of LDDs, the neuroimaging record shows additional involvement of the left posterior superior temporal (LPST) and lower parietal cortices, which are associated with Wernicke's aphasia. We test the hypothesis that this localization incongruence emerges from an interaction of memory and linguistic constraints involved in the real-time implementation of these dependencies and which had not been previously isolated. Capitalizing on a long-standing psycholinguistic understanding of LDDs as the workings of an active filler, we distinguish two linguistically defined mechanisms: GAP-search, triggered by the retrieval of the relative pronoun, and GAP-completion, triggered by the retrieval of the embedded verb. Each mechanism is hypothesized to have distinct memory demands and given their distinct linguistic import, potentially distinct brain correlates. Using fMRI, we isolate the two mechanisms by analyzing their relevant sentential segments as separate events. We manipulate LDD-presence/absence and GAP-search type (direct/indirect) reflecting the absence/presence of intervening islands. Results show a direct GAP-search-LIF cortex correlation that crucially excludes the LPST cortex. Notably, indirect GAP-search recruitment is confined to supplementary-motor and lower-parietal cortex indicating that GAP presence alone is not enough to engage predictive functions in the LIF cortex. Finally, GAP-completion shows recruitment implicating the dorsal pathway including: the supplementary motor cortex, left supramarginal cortex, precuneus, and anterior/dorsal cingulate. Altogether, the results are consistent with previous findings connecting GAP-search, as we define it, to the LIF cortex. They are not consistent with an involvement of the LPST cortex in any of the two mechanisms, and therefore support the view that the LPST cortex is not crucial to LDD implementation. Finally, results support neurocognitive architectures that involve the dorsal pathway in LDD resolution and that distinguish the memory commitments of the LIF cortex as sensitive to specific language-dependent constraints beyond phrase-structure building considerations.

Keywords: left inferior frontal cortex, Broca's and Wernicke's aphasia, supplementary motor area, precuneus, long-distance dependencies, sentence comprehension, working memory, attention 


\section{INTRODUCTION}

A long-distance or filler-gap dependency (LDD) is a syntacticosemantic relation between a pronominal element and a syntactically licensed position, or GAP, in an embedded clause. The LDD is thus the linguistic device that allows the pronominal element to be interpreted within the embedded clause. In the English sentence "The captain ${ }_{k}\left[w_{h} o_{k / j}\right.$ the sailor predicted that the weather would frighten $\left.(G A P)_{j}\right]$ smiled." the LDD is the connection between the relative pronoun and the object position of frighten, to which the semantic role of frightenee is assigned. LDDs have traditionally provided a window to explore the interaction between lexico-semantic and syntactic mechanisms involved in sentence composition, and have thus represented a rich space for neurolinguistic and psycholinguistic investigation. In LDDs, these mechanisms are specifically observed in the interpretation of the relative pronoun both as the object of the embedded verb (e.g., the frightenee) and as the coreferent to the head noun antecedent (e.g., The captain), mechanisms that are presumably grounded not only in fundamental properties of sentence composition such as argument structure licensing and discourse linking but also in the neurological properties of the linguistic subsystems that support those properties (e.g., Frazier et al., 1983; Frazier and Clifton, 1989; Grodzinsky, 1989; Swinney et al., 1989; Swinney and Zurif, 1995; Gibson, 1998; Grodzinsky, 2000; Phillips, 2003; Avrutin, 2006).

From a neurolinguistic perspective, LDD implementation also allows us to investigate how the interaction between sentence composition and memory should be understood, as well as what the cortical distribution of this interaction should be. Interpretation of the relative pronoun is, after all, expected to place significant demands on the memory system: the pronoun must be held in memory while the intervening syntactic and semantic material is parsed (in the present case "that the weather would"). The presence of intervening material taxes the processing system (e.g., King and Kutas, 1995; Cooke et al., 2002; Fiebach et al., 2002; Santi and Grodzinsky, 2012; Santi et al., 2015) and is subject to aging effects (Zurif et al., 1995). So, understanding the cortical distribution of these dependencies gives us insight into the basic commitments that any neurocognitive model of language must allow with respect to sentence composition in addition to the interactions of sentence composition with other components of cognition, most notably memory.

The record on LDD comprehension reveals a long-standing incongruence regarding the language processing commitments of the left inferior frontal (LIF) cortex: lesion studies show that in contrast to Wernicke's patients and patients with lesions in the right hemisphere homolog of Broca's area, Broca's patients fail to implement LDDs in a normal fashion during real-time comprehension. Specifically, these subjects fail to show normal implementation of the "GAP-filling" effect: the reactivation of the antecedent (i.e., the entity coreferent with the relative pronoun) at the position of the GAP (e.g., Zurif et al., 1993; Swinney et al., 1996; Grodzinsky et al., 1999; Grodzinsky, 2000; Burkhardt et al., 2003; Love et al., 2008). Given the localization value of Broca's and Wernicke's aphasia, this pattern of performance is taken to indicate that LDDs demand the workings of the LIF cortex and, crucially, do not depend on the workings of the left posterior superior temporal (LPST) cortex. By contrast, neuroimaging work has shown equal engagement of the LIF cortex and the LPST cortex for the implementation of the same dependencies (e.g., Stromswold et al., 1996; Cooke et al., 2002; Fiebach et al., 2002; Ben-Shachar et al., 2003, 2004; Friederici et al., 2003; Grodzinsky and Friederici, 2006; Santi and Grodzinsky, 2008).

We take both sets of results- lesion- and neuroimagingbased- to be valid and on that basis propose that together they provide complementary observations about LDDs and the neurocognitive resources that support them. Specifically, we hypothesize that one crucial property of LDD implementationGAP-search-relies on the workings of the LIF cortex, as the lesion-based record shows. This leaves open the question of the role of the LPST cortex reported in the neuroimaging record. In this respect we test the hypothesis that such LPST cortical recruitment would not be connectable to the implementation of GAP-search; and may be instead implicated in GAP-completion, a local, lexically-driven process fundamental to all sentence composition. To this end, we isolate the neurocognitive factors underpinning LDD comprehension on the basis of an analysis of relative pronouns that connects to parallel, incremental left-to-right structure-building mechanisms with potential neurocognitive relevance. Using fMRI, we examine the timing and cortical commitments of the interaction of these mechanisms. We conclude with a discussion of the implications of these findings for the lesion vs. imaging "mismatch," and in the context of current neurocognitive models for our understanding of the LIF cortex as a "language" area.

\subsection{The Structural and Processing Properties of Long-Distance Dependencies}

The purpose of this section is to present the linguistic structure for long-distance dependencies (LDDs) that supports their realtime processing implementation. This structure is therefore the basis for the definitions of the processing mechanisms of GAP-search and GAP-completion, which operationalize the dependency in neurocognitive terms ${ }^{1}$. In English, long-distance dependencies prototypically emerge in relative clause and whquestion formation. In the case of relative clauses, they involve three main elements: the antecedent, the relative pronoun, and the GAP. The antecedent is the denotation of the head noun

${ }^{1}$ This linguistic description captures the consensus among a variety of syntactic approaches, e.g., Government Binding/Minimalism, Head-Driven Phrase Structure Grammar, Lexical Functional Grammar, and Simpler Syntax, among others, that LDDs are grounded on two organizational properties of language: (1) the possibility to "package" the semantic and syntactic local conditions of the relative pronoun as lexicalized content in the form of subcategorization and/or selectional restrictions, and (2) the possibility of a GAP, a phonologically empty lexico-syntactic entity whose purpose is to instantiate the lexical requirements of the embedded verb; requirements that are expressed in the form of argument structure and subcategorization specifications. These are fundamental and widely accepted properties of the language system. The description presented here is therefore compatible with any representational analysis of relative pronouns that incorporates these two properties (see Culicover and Jackendoff, 2005, for extensive discussion of the syntax-semantics interactions in LDDs and the assumptions that lead the various approaches in question to favor one specific implementation over another). 
of the noun phrase containing the relative clause [captain in (1) below]. The RELPRO (which may be phonologically empty in English) is the entity that semantically links the antecedent and the GAP [who in (1) below]. The RELPRO occupies what we would call a "non-canonical" position, a position that does not receive direct semantic role assignment by a predicate, and therefore does not receive direct interpretation with respect to the proposition associated with the embedded clause. This interpretation is provided instead through the dependency it forms with the GAP. The GAP, in turn, is a hypothesized phonologically empty syntactically valid place-holder of the "displaced" relative pronoun which receives a semantic role by virtue of its grammatical function within the embedded clause. (1) below illustrates the relation between the GAP to which the semantic role of "experiencer" is assigned and the denotation of the head noun captain (the antecedent):

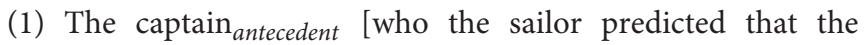
weather would frighten ("the captain") ${ }_{G A P}$ ] turned back to port.

The relation between the antecedent and the GAP is mediated by the relative pronoun (RELPRO). The RELPRO holds a coreference relation with the antecedent. And it is this coreference relation between the RELPRO and the antecedent that allows the antecedent to be interpreted as a participant in the proposition associated with the embedded clause, i.e., the sailor predicted that the weather would frighten the captain. Establishing an LDD therefore means connecting, on the one hand, the antecedent and the RELPRO and, on the other, the RELPRO and the GAP. These two distinct links are identified by the (shared) indices in (2) below:

(2) The captain $k$ [who $k / j$ the sailor predicted that [the weather would frighten $\left.(\mathrm{GAP})_{j}\right]$ ] turned back to port.

As can be seen, LDDs contain syntactic (construal of the relative pronoun as a grammatical relation in a "noncanonical" position) and lexico-semantic (semantic role assignment) mechanisms which are categorically distinct, and consequently subject to at least partially independent principles of composition. They also involve pronoun interpretation (the establishment of coreference between the RELPRO and the antecedent), which, at least for processing purposes, is identified as a discourse process (e.g., Grodzinsky et al., 1991; Avrutin, 1999; Piñango and Burkhardt, 2005). We take these mechanisms to be encoded in the lexical representation of the RELPRO itself as syntactic, discourse, and semantic selectional requirements respectively. The proposed representation is presented in (3) below:

$\begin{array}{rlll} & \{\text { Syn: } & {\left[{ }_{N P} \mathrm{~N}_{\text {head }}\right.} & {\left[{ }_{C P} \mathbf{N P}_{k / j / w}\right.} \\ \text { (3) RELPRO “who" } & \{\text { Sem: } & {\left[\text { argument }_{k}\right.} & \text { [pronoun }_{k / j / w} \\ & \{\text { Discourse: } & {\left[\text { antecedent }_{k}\right.} & {\left[\mathbf{P R O}_{k / j / w}\right.} \\ & \{\text { Phon: } & [\text { hu }]\} & \end{array}$

The representation in (3) specifies the syntactic, discourse, and lexico-semantic environments in which the RELPRO who may be licensed, thus capturing the main properties of its linguistic distribution in English. Retrieval of a RELPRO during comprehension therefore means the retrieval of this lexical composite with all the mutually constraining algorithms that determine the environment of its realization. In this way the lexical entry itself makes explicit the possible predictions by the parser regarding preceding and crucially, incoming lexical material.

This description thus represents the relevant lexicosyntactic characterization that we take to underlie both the filler-gap effect (e.g., Crain and Fodor, 1985; Stowe, 1986; Swinney et al., 1988; Frazier and Flores d'Arcais, 1989; MacDonald, 1989; McElree and Bever, 1989; Nicol and Swinney, 1989; Fodor, 1995) and its corresponding psycholinguistic generalization, the Active Filler Hypothesis (Frazier and Clifton, 1989). Specifically, in this linguistic articulation, the GAP is simply the realization of a coindexation relation between the relative pronoun and a phonologically unsupported [NP+semantic argument+grammatical relation] "triplet" in the embedded IP. The Active Filler Strategy therefore emerges as the implementation of the search to satisfy the RELPRO's requirements ${ }^{2}$. We conjecture that the explicitness of this lexically "packaged" parallel, multi-layer structure is what gives the LDD its seemingly unified processing implementation, what informs the parser as to the syntactic constituents where it can/cannot find a GAP (e.g., Stowe, 1986), and what so powerfully drives the RELPRO (the filler) to hypothesize a GAP even in constructions where it will ultimately be disallowed (e.g., Frazier et al., 1983; Hickok, 1993).

Having made explicit the necessary linguistic and psycholinguistic considerations, we turn to other non-linguistic real-time implementation requirements, specifically, memory requirements. We observe that there are in principle three "inflection points" in the LDD processing: the signaling by RELPRO retrieval that a GAP is incoming, the search for the GAP, and the actual instantiation of the GAP; that is, the point in the composition of the embedded clause where the RELPRO requirements are met (i.e., the GAP). We reason that whereas the antecedent-RELPRO coreference relation and GAP instantiation are unambiguous and local, the instantiation of the search for the GAP is, by contrast, multiply ambiguous due to the availability of multiple potential GAP positions that the RELPRO can be coindexed with and that are associated with all the possible grammatical relations in the embedded clause. This inherent ambiguity is presumably what forces the processor to closely track the syntactic and semantic structure of the incoming embedded clause until the GAP is reached, thus making it memory taxing. It is this basic difference what makes the gap search process a clearer candidate

$\begin{array}{ll}{\left[I P \mathrm{NP}_{j}\right.} & \left.\left.\left.\left.\left[\mathrm{V} \mathrm{NP}_{w}\right]\right]\right]\right]\right\} \\ \text { ppredicate }_{\text {SUBJ }_{j}} & \left.\left.\left.\left.\left[\operatorname{argument}_{j}, \text { argument }_{w}\right]\right]\right]\right]\right\} \\ \left.\left.\left.\left.\left[\mathrm{OBJ}_{w}\right]\right]\right]\right]\right\}\end{array}$

\footnotetext{
${ }^{2}$ The index alignment shown across constituents in the syntactic, semantic, and discourse layers makes explicit the observation by most linguistic frameworks of a robust correlation between syntactic category/position, grammatical relation, and
} 
for the probing of cortically localizable real-time linguistic processes.

On this basis, we articulate the LDD into two linguistically distinct stages, the search process itself vs. the licensing point of the GAP. These stages are in turn operationalizable as two mechanisms distinguishable by their differing memory demands. Those mechanisms are:

(i) GAP-search: triggered by the retrieval of the RELPRO. It is the language composition process where memory resources are maximally taxed: Upon retrieval of the RELPRO its lexico-syntactic requirements must be satisfied all while the phrase structure and semantic representations of the embedded-clause are being composed [e.g., who $o_{k / j}$ the sailor predicted that the weather, in (2)]. GAP-search is the mechanism that effectively implements the Active Filler Strategy: the RELPRO's lexically-driven search within the embedded clause in order to meet its lexico-syntactic requirements.

(ii) GAP-completion: triggered by the retrieval of the embedded verbal predicate. It is the process whereby the RELPRO's lexico-syntactic requirements are satisfied. In (2) this process takes place when the embedded verb is retrieved: the earliest point at which the embedded predicate (e.g., frighten) can license the object grammatical relation/NP structure and assign to the RELPRO the corresponding semantic role (e.g., frighten-ee). This not only completes the interpretation of the RELPRO who within the embedded clause but "grounds it," as it were, into the composition of the rest of the embedded clause. It allows the interpretation of the coreferring antecedent (the denotation of the matrix subject head, captain) as a participant in the embedded proposition's semantic representation. Crucially, this process, like GAPsearch, is compositional and therefore expected to require memory resources beyond lexical retrieval. However, given the locality of its resolution, the amount of memory resources GAP-completion demands should be significantly less than those demanded by GAP-search.

Here, we hypothesize that given their respective linguistic properties and correlated memory demands, these two mechanisms are potentially neurologically dissociable in a way that could shed light on the neurocognitive incongruence at issue. Notably, this kind of processing analysis finds direct support in previous findings by Phillips et al. (2005). That report presents two distinct electrophysiological components associated with long-distance dependency comprehension: a sustained anterior negativity subsequent to the initiation of the wh-dependency and a late posterior positivity (P600) associated with the completion of the dependency. We take that pattern to represent the electrophysiological correlates of GAP-search and GAP-completion respectively and thus take them as initial support for the analytical approach adopted here.

\footnotetext{
${ }^{2}$ semantic relation, such that if a predicate licenses, say, an agent argument, this argument will bear the subject function, which in English can be associated with NP category and SPEC;IP position (e.g., Chomsky, 1965, 1981; Bresnan, 1982, 2001; Fillmore, 1988; Goldberg, 1995; Van Valin and LaPolla, 1997; Culicover and Jackendoff, 2005).
}

Most crucially for our present purposes however, a closer look at the fMRI record also suggests the potential viability of this dissociation. We turn to that record directly below.

\subsection{LDDs and the LIF Cortex in fMRI: Previous Experimental Record}

In this section we discuss previous neuroimaging work that has also targeted either GAP-search or GAP-completion as we define them here in connection to the workings of the LIFG. Our search through the record was constrained by the requirement that the given report target one, the other, or both mechanisms in question as unified phenomena. The conclusions from that work together with the lesion-based evidence constitute the basis for the specific localizational predictions that we test ${ }^{3}$. Of the large body of neuroimaging work on LDD comprehension, four reports specifically deal with GAP-search as we have defined it: (Santi and Grodzinsky, 2007, 2010, 2012) and Matchin et al. (2014). Interestingly, we found no previous work on LDD comprehension targeting GAP-completion. In line with the focal lesion evidence these four reports converge on the observation that at least GAP-search, as we have defined it here, preferentially recruits the workings of the the LIF cortex. This is what unites them. In what follows we discuss for each of the reports the specifics of how these observations came to be.

Santi and Grodzinsky (2007) connect LDDs to the LIF cortex exclusively through what they call a "distance" effect. They test two phenomena. The one at issue involves object relatives in three conditions: one-NP embedded subject, two-NP embedded subject, and three-NP embedded subject. Crucially, these added NPs are irrelevant to the structure of the RELPROGAP dependency itself as the NPs have been added to the embedded subject phrase. Their function in the experimental design is to add material (specifically NP material which is syntactically identical to the RELPRO) between the RELPRO and the object-GAP. This material does not add to the complexity of the LDD but does increase the linear distance between the RELPRO and the GAP. In so doing, it increases the amount of structure the parser must build in order to get to the GAP. Such increase is coupled with an increase in number of nominals (one to three). Santi and Grodzinsky (2007)'s results show recruitment

\footnotetext{
${ }^{3}$ Our selectional criteria, necessary for our localizational purposes, had the unintended consequence of filtering out reports that have otherwise been valuable for our understanding of LDD processing. Fiebach et al. (2005), for example, connect (non-canonical) GAP-search to the LIF cortex, but report activation in other areas as well. For their Long $>$ Short (obj.) contrast, they report in addition to the LIF cortex, right inferior frontal (RIF) cortex, junction of the left precentral sulcus, bilateral STS, MTG (21/22) and the left thalamus. By contrast, for their Long > Short (subj.) contrast, no LIFG is reported. Instead, they report activation in the bilateral inferior portion and left superior portion of the parietooccipital sulcus (BA 17/30 and BA 7 respectively). So, this report relates the LIF cortex to GAP-search but not in a unified manner. Similarly, the results published in Makuuchi et al. (2009) address LDDs but are not directly relatable to our present objectives. Whereas they do report LIF cortex activation in connection to comprehension of double-center embedded clauses vs. single-embedded clauses akin to that reported by Santi and Grodzinsky (2007) and Fiebach et al. (2005), their report is based on a region of interest analysis exclusively, and not on a whole brain analysis. Whereas this approach makes sense given their specific interest in the internal articulation of the LIF cortex and not on localizing LDDs components, it prevents us from concluding whether the association they found targeted specifically the LIF cortex.
} 
of the LIF cortex in the three vs. two nominal increment. We see this manipulation as addressing GAP-search as we have defined it (to the exclusion of GAP-completion) because in the three-NP condition, the minimal difference was the increase in distance between the RELPRO and the GAP, and this greater distance had to be tracked in order for the parser to get to the GAP ${ }^{4}$.

More recently, Santi and Grodzinsky report in two separate papers, 2010 and 2012, an association between the LIF cortex and LDD processing which, given their respective designs, again target GAP-search to the exclusion of GAP-completion. Whereas in Santi and Grodzinsky (2010) the manipulation involves a comparison between GAP-search and embedding, connecting only GAP-search to the LIF cortex, Santi and Grodzinsky (2012) distinguishes general dependency from predictability, the ability of the parser to predict the need for a GAP. Their results show that predictability not dependency correlates with the LIF cortex effect, focused on BA $45^{5}$.

Finally, Matchin et al. (2014) test the hypothesis that the LIF cortex supports a more general "antecedent-variable" dependency function, thus allowing the possibility to consider GAP-search as a member of a larger family of "search"based processes. Such a hypothesis predicts an LIF cortex preferential activation for pronoun-antecedent relations (i.e., backward anaphora) which, like RELPRO-based LDDs, contain as a "variable" an element with an incomplete referential interpretation (pronoun) which must actively look for an "antecedent," the entity with which it must corefer. As with Santi and Grodzinsky (2007), the experimental design of Matchin

\footnotetext{
${ }^{4} \mathrm{We}$ do observe, though, that this association is not unambiguous. An alternative interpretation to these findings could be that the reported LIF cortex effect results instead from the composition of a more complex meaning structure associated with a semantically more informative embedded subject. In this scenario, preferential activation of the LIF cortex emerges not from greater LDD distance, but from the semantic demands of processing an incrementally more elaborate embedded subject in composition with the embedded transitive verb and its complement. Indeed, this kind of effect is connectable to a similar LIF cortex recruitment found by Husband et al. (2011) and Lai et al. (2014), who independently show LIF cortex involvement in connection, this time, to the processing of complement coercion (e.g., The girl began the book vs. The girl wrote the book), a phenomenon also described as involving "enrichment" of the semantic representation.

${ }^{5}$ In another related paper, Santi et al. (2015) test a distinction similar to the one reported in 2007. In addition to the NP category, they introduce CP as potential intervening category. Their results show that for both conditions together $(\mathrm{CP}+\mathrm{NP})$, there is, in addition to LIF cortex activation, RIF cortex activation, again correlated with distance. The novel comparison here is the joint results involving the $\mathrm{CP}$ condition which, as the authors point out, suggest that the syntactic category of the intervening material is not relevant to GAP-search, a conclusion that contrasts with previous findings regarding Broca's poor performance in $\mathrm{CP}$ production, and fMRI results showing $\mathrm{CP}$ processing in connection to the LIF cortex (Shetreet et al., 2009). As in the case of Santi and Grodzinsky (2007), we believe that their results warrant consideration of an alternative interpretation: the possibility that the increased cost contributed by the CP distance be due instead to the possible garden-path created by the absence of complementizer in the lower $\mathrm{CP}$. In the sentence "I knew [which porter the neurosurgeon said] $\mathrm{CP}_{2}$ [the resident liked GAP] $\mathrm{CP}_{1}$ " two possible structural paths are possible at $\mathrm{CP}_{2}$. Specifically, the $\mathrm{CP}_{2}$ verb "said" subcategorizes for both an NP and a CP. When an NP is suggested (due to the absence of the complementizer), the CP possibility is discarded. But this soon proves to be the wrong decision both on semantic grounds (the neurosurgeon said [the resident $]_{N P}$ ) and on syntactic grounds ( $*$ the neurosurgeon said [the resident liked $]_{* N P}$ ). Once the parser gets the lower verb "liked," it must revise its original decision in favor of the CP option, consequently incurring a cost.
}

et al. (2014) targets the GAP-search portion of the pronoundependency, as we have defined it. Their results show that only the subtractions involving backward anaphora (and not the RELPRO-based LDDs) yielded LIF cortex activation. And for these there was, in addition, activation in the right MTG, STC, bilateral SMA, bilateral occipital activation, and left STS. So, even though the observation is clearly made that the LIF cortex participates in predictive searches similar to GAP-search, it is also the case that other cortical regions also participate in this process, rendering the specific contribution of the LIF cortex in the processing of this kind of LDD inconclusive. This said, the presence of LIF cortex activation in this fairly different kind of dependency is suggestive of a deeper processing commonality, which so far has not been fully explored in the neuroimaging literature, and is one that we think may be captured by the generality of the GAP-search mechanism ${ }^{6}$.

In sum, whereas the vast majority of fMRI research involving LDDs correlate them to cortical regions beyond the LIF cortex, some do provide exclusive or close to exclusive correlation with LIF cortex. Those that do, target GAP-search as we have defined it. By contrast, GAP-completion, the other major LDD mechanism capturing the more general properties of LDD composition, remains less explored. In light of this, and in order to further understand the factors involved in the neurocognition of LDDs we ask the following questions: What is the neurocognitive relation between GAP-search and GAP-completion? Do they rely on the workings of overlapping brain regions? And, could we associate GAP-completion to the LPST cortex, thus directly addressing the lesion-neuroimaging incongruence? In addition, a new question is revealed: if the effects reported reflect GAPsearch, why are they observed mainly in the context of objectrelative GAPs? The specifics of the study seeking to address these question are presented directly below.

\subsection{The Study: Determining the Neurological Underpinnings of LDDs}

Our analysis above shows that LDD comprehension can be organized into at least two processing mechanisms. We propose here that the existence of this dual mechanism infrastructure and the differential memory resources that it demands is the source of the disparity regarding the cortical recruitment of LDD processing. Moreover, we propose that the reason it has not been detected before has been due to a limitation inherent to the traditional data-analysis approach used in the past. We thus propose that the cortical localizational incongruence is the result of the interaction of two factors: one linguistic and one methodological. The linguistic factor refers to the previous analyses which collapse GAP-search with RELPRO interpretation at the GAP position, GAP-completion, thus conflating processes with potentially distinct neurocognitive demands. The methodological factor refers to the traditional approach to data analysis in language-related fMRI whereby

\footnotetext{
${ }^{6} \mathrm{We}$ find this kind of comparison to be right-minded and useful also because it connects with independent work on the neurology of anaphora resolution which notably reports an impairment in pronoun and logophor resolution in Broca's patients (e.g., Grodzinsky et al., 1993; Avrutin, 1999; Pinango, 2003; Piñango and Burkhardt, 2005; Schumacher et al., 2010).
} 
subtractions take place at the sentence level, an approach which, in this case, prevents finer-grained exploration of the intrasentential components of the dependency.

We address the linguistic factor by testing constructions that vary the degrees of linguistic compositional demands and in doing so allow us to examine the two mechanisms separately. These compositional demands range from a condition where an LDD is not required, as in (4):

(4) The captain believed the sailor's prediction yesterday that the weather would frighten ${ }_{n o-g a p}$ the crew and turned back to port. (Condition D)

to one where an LDD is required and the link between the RELPRO and the GAP is syntactically direct, as in (2) above repeated here as (5):

(5) The $\operatorname{captain}_{k}\left[\mathbf{w h o} \mathbf{o}_{k / j}\right.$ the sailor predicted that the weather would frighten $(\mathrm{GAP})_{j}$ ] turned back to port. (Condition A)

to one where the syntactic connection between the RELPRO and the GAP is not direct [i.e., the intervening syntactic constituent does not contain the predicate licensing the GAP (6) $]^{7}$ :

(6) The $\operatorname{captain}_{k}\left[\mathbf{w h o} \mathbf{w}_{k / j}\right.$ [the sailor's prediction yesterday about the weather] had frightened ${ }_{g a p}$, turned back to port. (Condition $\mathrm{B} / \mathrm{C}$ )

Comparing these conditions allows us to observe the extent to which the memory-language interaction is sensitive to actual compositional linguistic mechanisms, and if so, which ones and with what cortical implications. In this respect, (5) > (4) and $(6)>(4)$ in particular allow us to assess the cortical resources that must be recruited as the processor actively searches for the GAP $[(5)>(4)]$ vs. those which must be recruited during the composition of sentence structure which the processor "knows" cannot contain a GAP, as in [(6) > (4)] (see Stowe, 1986; Kluender, 1998, respectively, for early evidence of the sensitivity of the processor to island constraints, and of how, and in contrast to widespread assumptions in linguistics,

\footnotetext{
"We call this condition "indirect GAP-search" and not "island" for the following reason: the term island refers to the perspective of the "moved" constituent before it has moved. This perspective states that such constituent cannot "leave" the larger constituent in which it is base-generated. To be sure, indirect GAP-search is a direct consequence of "movement"; but movement itself is only a metaphor, it has no processing status (i.e., the processor never carries out the movement; it only deals with its consequence). By contrast, the term indirect GAP-search is meant to refer to the perspective of the processor (left-to-right incremental composition). For the processor, what matters regarding any type of island is whether upon encountering a given constituent, it can hypothesize that the GAP is to be found within that constituent. If it can, then that constituent is searched for potential GAP positions, if it cannot, then the processor "waits", as it were, for that (local) constituent to end in order to continue the search. It is this situation that gives rise to the indirectness we refer to: the GAP is incoming, but not in the (minimal) constituent under construction. The "indirect GAP-search" label thus allows us to separate the linguistic intricacies of islands, which go well beyond the condition tested here, from one well-attested processing consequence of them. The label "indirect" therefore speaks to the fact that the subcategorized CP is not provided within the local constituent directly after the relative pronoun. So, from the perspective of the parser an "island" is simply a constituent that is not subcategorized and therefore it is not expected to contain the GAP.
}

islands could in fact result from the interaction of processing factors).

With these contrasts in place, we are able to discuss our approach to the examination of the role of memory in the longdistance dependency construction. We do this through a data analysis manipulation whereby the two hypothesized processing mechanisms, GAP-search and GAP-completion are analyzed as separate events. Specifically, we use an intra-sentential event-related subtraction approach whereby subtractions are performed over the relevant non-overlapping segments of the sentence (see Data Analysis section below for technical details). This, in combination with the minimal contrasts in the linguistic manipulation between conditions, presence/absence of GAP and presence/absence of direct antecedent-GAP link, allows us to isolate simple phrase-structure building from active GAP-search and from GAP-completion, respectively. The details of the experimental design and data analysis are presented directly below (see Lai et al., in press for a similar use of event-related design in the context of semantic composition).

\section{THE STUDY: INVESTIGATING GAP-SEARCH AND GAP-COMPLETION}

\subsection{Materials}

The study contained a total of four conditions (A, B, C, and D) with 60 sentences in each of the conditions. Sentences were constructed as matching quadruples, thus controlling for nonrelevant lexico-semantic and syntactic factors. This resulted in a final script of 240 sentences (60 quadruples). Test sentences for Conditions A and B were directly modeled from Gibson and Warren (2004), which introduces the \pm direct RELPROGAP link manipulation. A sample of a quadruple is presented in Table 1 below. As can be seen, whereas the conditions differ in the relevant syntactic properties (e.g., verbal vs. nominal: "sailor predicted" vs. "sailor's prediction") they share all other main lexico-semantic components, thus ensuring that they were as close as possible in terms of number of words, word frequency, and sense co-occurrence. Given our interest in separating activation related to GAP-search from that related to GAPcompletion our unit of analysis was the Event which was a segment of the sentence. Accordingly, condition matching had to be implemented especially at the event level. For matching (and data analysis) purposes then each sentence was construed in terms of three events which in Table 2 are observable in the internal bracketing of the sentences: Event $\mathbf{0}$ contains the material before the brackets including head noun and relative pronoun/verb, Event 1 corresponding to GAP-search contains the material in bold within brackets; and Event 2 corresponding to GAP-completion contains the material after the brackets. As can be seen, for Event 1, all conditions match in terms of number of words. For Event 2, condition D, the control condition has in addition three words corresponding to the object NP (two words) and the conjunction (one word). We note that as this is the control condition any extra activation associated with the three extra words would be eliminated in the subtraction process. 
TABLE 1 | Four experimental conditions.

\begin{tabular}{|c|c|c|}
\hline & Sentence & Condition \\
\hline A & $\begin{array}{l}\text { The captain, who [the sailor predicted yesterday that the weather] would frightengap,turned back } \\
\text { to port. }\end{array}$ & GAP-search/direct and GAP-completion \\
\hline $\mathrm{B}$ & $\begin{array}{l}\text { The captain, who [the sailor's prediction yesterday about the weather] had frightenedgap,turned } \\
\text { back to port. }\end{array}$ & GAP-search/indirect and GAP-completion \\
\hline C & $\begin{array}{l}\text { *The captain, who [the sailor's prediction yesterday about the weather] had frightened the crew, } \\
\text { turned back to port. }\end{array}$ & $G A P$-search/indirect and $G A P$-completion violation \\
\hline D & $\begin{array}{l}\text { The captain believed [the sailor's prediction yesterday that the weather] would frighten the crew } \\
\text { and turned back to port. }\end{array}$ & No GAP-search and No GAP-completion \\
\hline
\end{tabular}

TABLE 2 | Experimental conditions by events.

\begin{tabular}{|c|c|c|c|}
\hline Condition & Event 0 & Event 1 ( \pm GAP-search) & Event 2 ( \pm GAP-completion) \\
\hline A & The politician who & the journalist claimed that the government report & had botheredgap is calling a press conference \\
\hline$B$ & The politician who & [the journalist's claim about the government report $]_{i s l a n d}$ & had botheredgap is calling a press conference \\
\hline C & The politician who & [the journalist's claim about the government report] $]_{\text {island }}$ & had bothered the people is calling a press conference \\
\hline D & The politician believed & the journalist's claim that the government report & had bothered the people and is calling a press conference \\
\hline
\end{tabular}

TABLE 3 | Planned subtractions by events: single subtractions.

\begin{tabular}{|c|c|c|}
\hline Subtraction & Event 1 & Event 2 \\
\hline$A>D$ & GAP-search direct & GAP-completion \\
\hline$B>D$ & GAP-search indirect & \\
\hline$A>B$ & $\begin{array}{l}\text { GAP-search }(G A P \text {-search } \text { direct }- \\
\text { GAP-search } \\
\text { indirect })\end{array}$ & - \\
\hline$B>C$ & - & GAP-completion \\
\hline
\end{tabular}

(For further description of the analysis approach see Table 3 in the Data Analysis section).

Table 1 presents the conditions with their respective dependencies. Figure $S 1$ in the Supplementary materials presents the corresponding syntactic structures (Note that for Conditions $\mathrm{A}$ vs. $\mathrm{B} / \mathrm{C}$, the different syntactic structures determine the nature of the link between the RELPRO and GAP: direct for $\mathrm{A}$ and indirect for $\mathrm{B})$. Asterisk $(*)$ in Condition C signals ungrammaticality.

In addition, the $\mathrm{A}, \mathrm{B}$, and $\mathrm{D}$ conditions were pre-tested for acceptability using a five-point likert scale. This pre-test allowed us to ensure that even though $\mathrm{D}$ would be more acceptable than $\mathrm{A}$ and $\mathrm{B}$, there would be no difference in acceptability between $\mathrm{A}$ and $\mathrm{B}$ conditions. And this is what planned comparisons show. As expected Condition $\mathrm{D}\left[\mathrm{D}_{\text {mean }}=3.79(S D=0.5)\right]$ was deemed significantly more acceptable than conditions $\mathrm{A}\left[\mathrm{A}_{\text {mean }}=2.66\right.$ $(S D=0.5)(t=-4.05, p<0.001)]$ and B $\left[B_{\text {mean }}=2.67(S D=0.6)\right.$ $(t=-4.1, p<0.001)]$. Also as expected no statistical difference in acceptability between A and B was found $(t=-0.03, p=0.48)$. This was calculated on the basis of responses from a sample of 13 native English speakers from the Yale undergraduate population, the same population from which the fMRI participants were selected.

Comprehension questions followed all condition $\mathrm{A}, \mathrm{B}$, and $\mathrm{D}$ sentences. No questions followed condition $\mathrm{C}$ sentences as the kind of ungrammaticality in that condition makes it difficult to ask questions that have an unambiguous yes/no answer. This said, we note that the ungrammaticality in Condition $\mathrm{C}$ appears toward the end-of the sentence, crucially, at the GAP-completion segment sentence. So, subjects could not know during the first part of the sentence up to the embedded verb whether they were in the presence of a grammatical or ungrammatical sentence. This motivated them to pay attention to all sentences equally.

In addition, questions probed different combinations of the matrix subject, embedded subject, matrix verb, and embedded verb. This variability was introduced intentionally to motivate participants to pay attention throughout the sentence as opposed to specific features of the sentence. To further minimize strategizing, the assignment of a given question to a given sentence was random, so even if the participants could realize that the matrix/embedded subject nouns and the matrix/embedded verbs mattered, for any given sentence they could not predict what specific element would be queried. So, they had to pay attention to all components of the sentences equally. For a sentence like The captain, who the sailor predicted yesterday that the weather would frighten, turned back toward port., subjects would get one of these possible questions:

i. Did the sailor predict that the weather would frighten the captain? (expected answer: Y)

ii. Did the captain predict that the weather would frighten the sailor? (expected answer: N)

iii. Did the captain turn back toward port? (expected answer: $\mathrm{Y}$ ) iv. Did the sailor turn back toward port? (expected answer: N)

Coming back to the experimental sentences, this is what each condition probes:

Condition A examines GAP-search, triggered at who and GAP-completion. The distance between the RELPRO and the GAP is expected to reveal the workings of the memory system in a situation where finding the GAP is expected, given the 
absence of intervening islands, as compared to Condition D, the no-GAP condition, and Condition $\mathrm{B}$, the island condition where the GAP is not expected within the local constituent. Condition B also combines GAP-search (triggered at who) and GAP-completion. However, in contrast to Condition $A$, in Condition $B$ the search for the GAP must bypass the embedded subject (which is an island). Bypassing the embedded subject means that the processor needs to wait for that NP constituent to end to find the GAP. That is what the $\mathrm{B}>\mathrm{D}$ contrast is intended to reveal.

For both $\mathrm{A}>\mathrm{D}$ and $\mathrm{B}>\mathrm{D}$ contrasts there is a clear interaction with the memory system in connection to GAP-search. So similarity in recruitment is expected. A difference in recruitment $(A>D)$ and $(\mathrm{B}>\mathrm{D})$ would then be interpreted as a difference in the quality of the interaction with respect to GAP-search, one where the processor is not actively looking for the GAP $(B>D)$, vs. one where it is $(A>D)$.

Condition C is identical to Condition B except that the GAP position has been filled with an additional NP, which renders the sentence ungrammatical. The motivation for this condition focuses on the possible distinct cortical recruitment associated with GAP-completion. If, as we hypothesize, GAPcompletion has distinct neurological commitments from GAPsearch, this process will be observed as a unique activation pattern when comparing Conditions $\mathrm{B}>\mathrm{C}$, as these two conditions differ only with respect to the GAP-completion factor. $\mathrm{B}>\mathrm{C}$ thus effectively brings us the closest to observing the preferential recruitment for GAP-completion alone.

Condition D represents the control condition. It has the same number of words and constituents as the Condition $\mathrm{A}$ and $B$ counterparts, thus equally requiring full phrase-structure building and semantic composition. It lacks a long-distance dependency, so it is expected to tax the memory system the least in comparison to Conditions A or B.

\subsection{Design}

Each subject was presented with the 240-sentence script containing the 4 conditions, A, B, C, and D (60 items per condition). No additional fillers were included in the script. All 240 sentences were distributed in a pseudo-random fashion in 10 separate runs of 24 sentences each. The four experimental conditions were distributed in a counterbalanced fashion within each run such that no two sentences of the same quadruple would be included in the same run. Each subject was presented with a unique order of runs. So, in the end no two subjects saw the exact same sentence presentation order.

Each sentence presented had a maximum of 22 words. Each word in the sentence was visually presented at $500 \mathrm{~ms}$ per word. The $500 \mathrm{~ms} /$ word pace was chosen out of a variety of timings previously considered because it was the one that optimized ease of reading, speed, and accuracy in the comprehension of the sentence.

For $180(75 \%)$ of the sentences, a query (yes/no question) about the sentence just read was presented for $4000 \mathrm{~ms}$. The ISIs within and between (sentence+query) items were each $500 \mathrm{~ms}$ for a total of $16 \mathrm{~s}$ per item. Accordingly, the total time per run was $6 \mathrm{~min} 24 \mathrm{~s}$ ( $16 \mathrm{~s} \times 24$ sentences).

\subsection{Procedure}

The pre-scanning practice session was designed to familiarize the participants not only with the general procedure in the scanner but also with the length of the experimental sentences. In this practice session each participant was exposed to long embedded sentences similar to the ones they would be encountering in the study and at the same reading pace: one word at a time, paced at $500 \mathrm{~ms}$ per word, presented at the center of the screen and followed by a comprehension question.

Participants were instructed to read the sentences silently in the most natural way possible. To facilitate this, sentences were presented with punctuation marks (commas) supporting a native prosodic contour. Responses to the queried sentences were recorded with a yes/no button box. The total duration of the functional component of the study was about an hour, and the total duration of the testing session was $90 \mathrm{~min}$.

\subsection{Participants}

Fifteen native speakers of English ( 8 female and 7 male) between the ages of 18 and 22 participated in this study. All except for one subject were right handed with normal or correctedto-normal vision. By their own report, none had suffered a concussion nor were they under treatment for a neurological or psychological condition. All participants gave written informed consent in accordance with the guidelines set by the Yale University Human Subjects Committee and were compensated for their participation.

\subsection{Data Acquisition}

Head positioning in the magnet was standardized using the canthomeatal landmarks. In the scanner, cushions inside the head coil were used to reduce head movement and headphones were used to dampen the scanner noise and to communicate with participants. Conventional T1-weighted spin-echo sagittal anatomical images were acquired for slice localization using a $1.5 \mathrm{~T}$ whole body imaging system with a quadrature head coil (Siemens, Erlangen, Germany). After a 3-plane localizer and a multiple-slice sagittal localizer, $28 \mathrm{~T}-1$ weighted axial slices $(T R=485 \mathrm{~ms} ; T E=11 \mathrm{~ms}$; bandwidth $=130 \mathrm{~Hz} /$ pixel; $F A=$ $90^{\circ}$; slice thickness $=5 \mathrm{~mm}$; FOV $=200 \times 200 \mathrm{~mm}$; matrix $=256 \times 256)$ were obtained using flash spin-echo imaging parallel to the anterior and posterior commissure (AC-PC). Ten functional data series were then acquired with a single-shot gradient-echo echo planar imaging (EPI) sequence $(T R=2000$ $\mathrm{ms} ; T E=30 \mathrm{~ms}$; bandwidth $=1735 \mathrm{~Hz} /$ pixel; $F A=80^{\circ}$; slice thickness $=5 \mathrm{~mm}$; FOV $=220 \times 220 \mathrm{~mm}$; matrix $=64 \times 64$; with 196 measurements) with same slice localizations as the T1 anatomical. Stimuli were projected onto a semi-transparent screen at the head of the bore, viewed by the subject via a mirror mounted on the head coil. At the end of the functional imaging, a high resolution 3D Magnetization Prepared Rapid Gradient Echo (MPRAGE) sequence $(T R=24 \mathrm{~ms} ; T E=4.66 \mathrm{~ms}$; bandwidth $=$ $130 \mathrm{~Hz} /$ pixel; $F A=45^{\circ}$; slice thickness $=1.3 \mathrm{~mm}$; FOV $=340 \times$ 
$340 \mathrm{~mm}$; matrix $=256 \times 256)$ was used to acquire sagittal images for multi-subject registration.

\subsection{Data Analysis}

All data were converted from Digital Imaging and Communication in Medicine (DICOM) format to analyze format using XMedCon (Nolfe et al., 2003). During the conversion process, the first three images at the beginning of each of the eight functional series were discarded to enable the signal to achieve steady-state equilibrium between radio frequency pulsing and relaxation leaving 193 images per slice per trial for analysis. Functional images were realigned (motion-corrected) with the Statistical Parametric Mapping 5 algorithm (www.fil.ion.ucl.ac.uk/spm/software/spm5) for three translational directions $(\mathrm{x}, \mathrm{y}$, or $\mathrm{z})$ and three possible rotations (pitch, yaw or roll). Trials with linear motion that had a displacement in excess of $1.5 \mathrm{~mm}$ or rotation in excess of 2 degrees were rejected.

Individual subject data were analyzed using a General Linear Model (GLM) on each voxel in the entire brain volume with regressors specific for each task. For each of the four sentence types (A, B, C, D) there were four regressors (shown in Table 2): Event $\mathbf{0}=$ onset of the first word up to the offset of "that/about," Event 1, GAP-search = onset of subject of relative/complement clause up to offset of word before lowest embedded verb; Event 2, GAP-completion $=$ onset of lowest embedded verb up to end of the sentence, Question = onset of comprehension question up to the end of the question. We account for the hemodynamic delay within the General Linear Model used which includes the waver hemodynamic response function (hrf) from the AFNI software.

The resulting beta images for each task were spatially smoothed with a $6 \mathrm{~mm}$ Gaussian kernel to account for variations in the location of activation across subjects. The output maps were normalized beta-maps, which were in the acquired space $(3.438 \times 3.438 \times 5 \mathrm{~mm})$.

To take these data into a common reference space, three registrations were calculated within the Yale BioImage Suite software package (www.bioimagesuite.org, Papademetris et al., 2006). The first registration performs a linear registration between the individual subject raw functional image and that subject's $2 \mathrm{D}$ anatomical image. The $2 \mathrm{D}$ anatomical image is then linearly registered to the individual's $3 \mathrm{D}$ anatomical image. The $3 \mathrm{D}$ differs from the $2 \mathrm{D}$ in that it has a $1 \times 1 \times 1 \mathrm{~mm}$ resolution whereas the $2 \mathrm{D} z$-dimension is set by slice-thickness and its $\mathrm{x}-\mathrm{y}$ dimensions are set by voxel size. Finally, a non-linear registration is computed between the individual $3 \mathrm{D}$ anatomical image and a reference $3 \mathrm{D}$ image. The reference brain used was the Colin27 Brain (Holmes et al., 1998) which is in Montreal Neurological Institute (MNI) space (Evans et al., 1992) and is commonly applied in SPM and other software packages. All three registrations were applied sequentially to the individual normalized beta-maps to bring all data into the common reference space.

Data were corrected for multiple comparisons by spatial extent of contiguous suprathresholded individual voxels at an experiment-wise $p<0.05$. In a Monte Carlo simulation within the AFNI software package and using a smoothing kernel of
$6 \mathrm{~mm}$ and a connection radius of $6.97 \mathrm{~mm}$ on $3.44 \times 3.44 \times$ $5 \mathrm{~mm}$ voxels, it was determined that an activation volume of 197 original voxels (5319 microliters) satisfied the $p<0.05$ threshold. Clusters were created for each of the four subtractions. Each cluster was identified with a region label, and then associated with additional numeral labels corresponding to Brodmann areas. Regional labels were assigned using the Yale Brodmann Area Atlas which is defined on the Colin27 Brain at $1 \mathrm{~mm}$ resolution.

\subsection{Predictions}

Table 3 presents the planned single subtractions isolating the two mechanisms in question and corresponding to the two (intrasentential) events: Event 1 and Event 2. Event 1-related subtractions target GAP-search and direct vs. indirect GAP-search: the correlates of a lexically driven search for the GAP in two contexts, direct vs. indirect, above and beyond phrase-structure building considerations. Event 2-related subtractions target GAPcompletion: the satisfaction of the syntactic and lexico-semantic requirements of the RELPRO as comprehension unfolds. In addition, a series of double subtractions and three conjunction analyses were also performed to show whether or not any of the potential effects observed could be viewed as tapping a common cognitive process and if so which one. The specific double subtractions and conjunction analyses are presented further below in connection to the corresponding general predictions ${ }^{8}$.

If GAP-search-which takes place during Event 1 - and GAP-completion-which takes place during Event 2- place compositionally distinct linguistic demands with presumably different memory load implications, then they are likely to have distinct cortical recruitment commitments. The existence of distinct cortical recruitment is in turn hypothesized to be the root of the lesion-based/neuroimaging incongruence regarding LDD implementation. This distinction in recruitment should be observed between the two events across the relevant conditions (e.g., Conditions A and B vs. Condition D during Event 1 and Conditions A and D vs. Condition D during Event 2). Specifically:

\subsubsection{Prediction for GAP-search: GAP-search direct and $G A P$-search indirect}

If the LIF cortex supports GAP-search, regardless of whether it locally leads to a GAP position or not, both the GAP-search direct and GAP-search indirect $_{\text {conditions (Condition A, Event } 1 \text { and }}$ Condition B, Event 1, respectively) should elicit the same pattern when a no-GAP condition is subtracted (Condition D, Event 1).

If, by contrast, the brain distinguishes between the situation where the memory system is actively participating in the GAPsearch process, rather than simply supporting the phrase structure composition that happens to involve this process, we should observe a divergence in activation. In this case we expect that at least GAP-search direct - the condition that has been previously reported to be vulnerable in Broca's aphasia, is correlated with LIF cortex activation.

\footnotetext{
${ }^{8}$ We thank a reviewer for calling our attention to the importance of these two second-order analyses which as will be seen strengthened the quality of the evidence overall.
} 
Three double subtraction analyses (1) GAP-search $\mathrm{A}_{1}>\mathrm{D}_{1}$ vs. GAP-completion $\mathrm{A}_{2}>\mathrm{D}_{2}$, (2) GAP-search $\mathrm{B}_{1}>\mathrm{D}_{1}$ vs. GAP-completion $\mathrm{B}_{2}>\mathrm{D}_{2}$ and (3) GAP-search direct $\mathrm{A}_{1}>\mathrm{D}_{1}$ vs. $G A P$-search indirect $\mathrm{B}_{1}>\mathrm{D}_{1}$ and one conjunction analysis GAPsearch direct $\mathrm{A}_{1}>\mathrm{D}_{1}$ and GAP-search indirect $_{\mathrm{B}_{1}}>\mathrm{D}_{1}$ are relevant for this prediction. The first two double subtractions test LIF cortex sensitivity to GAP-search once activation associated with GAP-completion has been eliminated. The third double subtraction and the conjunction analysis allows us to see the extent to which GAP-search direct $_{\text {and }}$ GAP-search indirect $_{\text {have }}$ common activation.

\subsubsection{Prediction for GAP-completion}

Our analysis confers GAP-completion a subordinate role in LDD composition as it is a strictly local process connecting GAP-search to the ongoing composition of the sentence. In terms of cortical localization, we have seen that the previous neuroimaging record does not isolate it. By contrast, the focal-lesion record gives us an important clue as to GAP-completion's potential cortical distribution: For Broca's patients, the reactivation of the GAP, presumably involving GAP-completion, is not simply absent, it is abnormal. The GAP-filling effect is absent right after the licensing verb, but visible around $500 \mathrm{~ms}$ later (e.g., Burkhardt et al., 2003; Love et al., 2008). On the basis of our analysis, we interpret this comprehension pattern as the manifestation of a dissociation between GAP-search and GAP-completion such that the latter is evidently impacted by, but is not crucially dependent on, the workings of the LIF cortex.

Completing this picture, the lesion-based evidence also tells us that Wernicke's patients are able to implement gap-filling in a timely manner. Yet, in offline tasks such as sentence-to-picture matching, these very patients show impaired comprehension not only of object relative clauses, but also of subject relative clauses and non-embedded agentive matrix clauses, a behavior that has traditionally been rooted to a lexically-based deficit, and that accordingly confers Wernicke's area a generalized compositional role with direct semantic implications (e.g., Caramazza and Zurif, 1976; Shapiro and Levine, 1990; Piñango and Zurif, 2001, 2015).

Combining these pieces we reason that if there is a connection between LDD composition and the LPST cortex at all, it should be neither in connection to GAP-search nor to GAP-completion specifically, but in connection to a more general compositional process, involving the coupling of morphosyntactic and semantic composition, of which GAP-completion is but one manifestation. So, the localization prediction for GAP-completion is exploratory: GAP-completion-targeted in three Event 2-related comparisons (1) Condition B vs. Condition C, (2) Condition B vs. Condition D, and (3) Condition A vs. Condition D-should not activate the LIF nor the LPST cortices. But it should show an activation pattern that is instead neuroanatomically connectable to both LIF and LPST cortex associated with Broca's and Wernicke's aphasia respectively.

In terms of the double subtraction and conjunction analyses associated with this prediction, our objective is to determine whether or not, despite arising from different contrasts, the three activation patterns predicted to reveal GAP-completion indeed manifest the same preferential recruitment. Specifically, we compare $A_{2}>D_{2}$ and $B_{2}>D_{2}$ to each other and crucially to $\mathrm{B}_{2}>\mathrm{C}_{2}$, and look at how they differ (subtractions) and what cortical recruitment they have in common (conjunction).

In the strongest form of the prediction, if $A_{2}>D_{2}$ and $B_{2}>D_{2}$ are targeting the same process, subtracting them from each other and from $B_{2}>C_{2}$ should result in no difference. By the same token if the three subtractions are revealing the same cognitive process, the conjunction analysis with all three subtractions $A_{2}>D_{2}$ and $B_{2}>D_{2}$ and $B_{2}>C_{2}$ should show a high degree in overlap, one that is coherent with the single subtraction results.

\section{RESULTS}

\subsection{Behavioral Task}

Results from the post-sentential questions show an average accuracy rate of $87.6 \%$, which was distributed across conditions as follows: Condition A: $90.57 \%$ (29.24), Condition B: $87.47 \%$ (33.1), Condition D: $84.82 \%$ (35.9). A mixed-model analysis revealed a marginally significant effect of condition [Chi-square $=7.59,(d f=2) p=0.083]$. Pairwise comparisons revealed a significant difference in A vs. D $(p=0.001)$ and a marginally significant difference in A vs. B $(p=0.08)$. There was no difference between $\mathrm{B}$ vs. $\mathrm{D}(p=0.1)$ (all results corrected for multiple comparisons).

Given that all conditions show an accuracy rate higher than $80 \%$, we interpret the A vs. D difference as the result of lapses in attention due to the relatively undemanding nature of the $\mathrm{D}$ condition which allowed the subjects to lose concentration and in turn miss some of the comprehension questions.

\subsection{Isolating GAP-search: GAP-search direct and GAP-search indirect $_{\text {in }}$}

Figure 1A shows the pattern of activation, presented in radiological format, for the $A_{1}>D_{1}$ subtraction (Conditions $A$ and $\mathrm{D}$, Event 1). Two main regions of preferential activation are observed: the first one involves left BAs 45, 44, 47, 22 (inferior, medial), 38, and insula. The second one involves posterior cingulate, left primary and association cortex, and BAs 7 and 31 (both bilateral). Figure $1 B$ shows the $B_{1}>D_{1}$ contrast. Interestingly, this pattern of activation appears as a non-overlapping recruitment involving one region connecting bilateral BA 6 (medial superior), bilateral BA 8, and bilateral BA 32 and right BA 24. Table 4 below shows the significant differential volume by region for each of these comparisons.

The first double subtraction $\left(A_{1}>D_{1}\right.$ vs. $\left.A_{2}>D_{2}\right)$ (Figure 2, Table 5 below) shows a pattern almost identical to the one yielded by the original single subtraction: left BAs $47,46,45,44$, and 38 , medial BA 7, BAs 17, 18, and 19, and the cerebellum. The second and third double subtractions $\left(B_{1}>D_{1}\right.$ vs. $\left.B_{2}>D_{2}\right)$ and $\left(A_{1}>D_{1}\right.$ vs. $\left.B_{1}>D_{1}\right)$ by contrast yielded no significant activation.

Finally, the conjunction analysis counterpart comparing direct vs. indirect search showed an empty intersect. This analysis which, crucially, is based only on the corrected maps, tells us that for this comparison the stronger more reliable activation is in terms of the differences in preferential activation between the two contrasts. This supports the possibility that for Event 1, any privileged association is not between LIF cortex and GAP-search but between LIF cortex and GAP-search direct $_{\text {. }}$ 

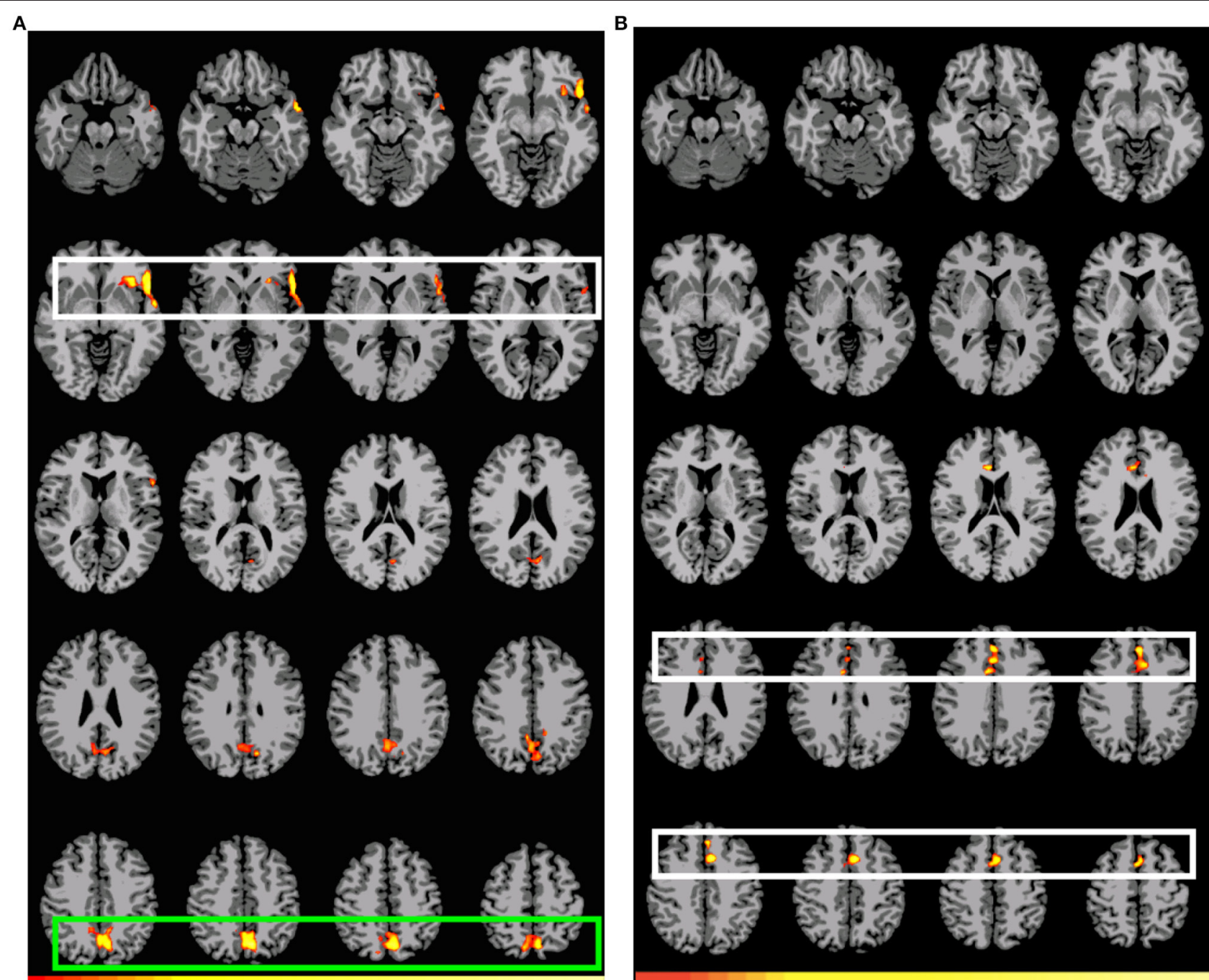

FIGURE 1 | Preferential activation for both GAP-search (Event 1) subtractions. Images are shown corrected at $p<0.05$ in radiological format (LH is on the right). (A) (Direct) GAP-search (Event 1) subtraction: $A_{1}>D_{1}$ : "the journalist claimed that the government report" > "the journalist's claim that the government report." White: BAs 45, 44, and 47. Green: Posterior cingulate and sensory association cortex. Low thresholds $(p<0.05 ; t=2.14)$ are indicated by red, while high thresholds $(p<0.000007$; $t=6.94)$ are indicated by yellow. (B) (Indirect) GAP-search (Event 1) subtraction: $B_{1}>D_{1}$ : "the journalist's claim about the government report" > "the journalist's claim that the government report." White: SMA activation. Only positive activation reported. Low thresholds $(p<0.05 ; t=2.14)$ are indicated by red, while high thresholds $(p<0.00002 ; t=6.42)$ are indicated by yellow.

\subsection{Isolating GAP-completion}

Table 6 (Figure 3) shows the pattern of activation for all three contrasts involving GAP-completion: Condition B > Condition C (Event 2), Condition A > Condition D (Event 2), and Condition $\mathbf{B}>$ Condition $\mathbf{D}$ (Event 2), respectively. We interpret them together because the pattern of activation they each give rise to is by our hypothesis reflecting the same GAPcompletion process. We present them separately because each emerges from different surface-level subtractions: a legitimately filled gap vs. an illegitimately filled gap $\left(\mathrm{B}_{2}>\mathrm{C}_{2}\right)$ and a filled gap vs. non-gap $\left(A_{2}>D_{2}\right.$ and $\left.B_{2}>D_{2}\right) .{ }^{9}$ Moreover, those segments

${ }^{9} \mathrm{~A}$ reviewer asks us about the meaningfulness of the $\mathrm{B}>\mathrm{C}$ (event 2) comparison. The results from the $\mathrm{B}>\mathrm{C}$ (event 2) subtraction reflect the preferential activation come from different (non-local) sentential contexts ( $\mathrm{A}$ and $\mathrm{B}$, respectively). We reason that if GAP-completion is an isolable process, it should yield a similar activation pattern regardless

triggered by $\mathrm{B}$ (event 2) without the material that is represented by $\mathrm{C}$ (event 2). We infer that this preferential activation includes any process involved in the interpretation of the GAP that survives the violation which, by our analysis, includes the interpretation of the GAP by means of the dependency formation. Indeed, we take this to be the substance of the residual of the subtraction. This contrasts with the violation condition, where the GAP position has been independently filled thus preventing dependency from being completed. As we discuss here, what is interesting of this subtraction, $\mathrm{B}>\mathrm{C}$ (event 2), is that despite emerging from a subtraction by a violation, the resulting pattern is comparable to the others in Event 2, particularly in terms of the SMA and parietal activation pattern, which the other two non-violation based contrasts also show. 
TABLE 4 | Significant differential volumes by region for GAP-search subtractions.

(Direct) GAP-search (Event 1) subtraction, $A_{1}>D_{1}$

\begin{tabular}{|c|c|c|c|c|}
\hline Region & Volume $\left(\mathrm{mm}^{3}\right)$ & Mean $T$-value & Max $T$-value & Max MNI Coords. (x, y, z) \\
\hline $\begin{array}{l}\text { Region 1: left BA 45, left BA 44, left BA 47, left BA } \\
22 \text { (inferior, medial), left BA 38, insula }\end{array}$ & 5571 & 2.94 & 5.83 & $-54,18,-3$ \\
\hline $\begin{array}{l}\text { Region 2: posterior cingulate, left visual cortex } \\
\text { (primary and association), bilateral BA } 7 \text { and } 31\end{array}$ & 9516 & 2.82 & 6.94 & $-9,-66,45$ \\
\hline
\end{tabular}

(Indirect) GAP-search (Event 1) subtraction, $B_{1}>D_{1}$

\begin{tabular}{|c|c|c|c|c|}
\hline Region & Volume $\left(\mathrm{mm}^{3}\right)$ & Mean $T$-value & Max $T$-value & Max MNI Coords. (x, y, z) \\
\hline $\begin{array}{l}\text { Right BA 24, bilateral BA } 6 \text { (medial superior), } \\
\text { bilateral BA 8, bilateral BA } 32\end{array}$ & 6262 & 2.80 & 5.56 & $-6,15,45$ \\
\hline
\end{tabular}

TABLE 5 | Significant differential volumes by region for GAP-search (Event 1) double subtraction $\left(A_{1}>D_{1}\right)$ vs. $\left(A_{2}>D_{2}\right)$.

\begin{tabular}{|c|c|c|c|c|}
\hline Region & $\begin{array}{c}\text { Volume } \\
\left(\mathrm{mm}^{3}\right)\end{array}$ & $\begin{array}{c}\text { Mean } \\
T \text {-value }\end{array}$ & $\begin{array}{c}\text { Max } \\
T \text {-value }\end{array}$ & $\begin{array}{c}\text { Max MNI } \\
\text { Coords. }(x, y, z)\end{array}$ \\
\hline $\begin{array}{l}\text { Region } 1 \text { left BA 47, left BA 46, } \\
\text { left BA 45, left BA 44, left BA } 38 \\
\text { (temporal pole) }\end{array}$ & 9328 & 2.70 & 6.03 & $-51,30,-6$ \\
\hline $\begin{array}{l}\text { Region 2: posterior cingulate, } \\
\text { sensory (association) }\end{array}$ & 7522 & 2.94 & 5.84 & $0,-63,36$ \\
\hline Region 3: cerebellum & 7526 & 2.71 & 6.15 & $24,-69,-33$ \\
\hline
\end{tabular}

of non-local context. This is especially the case for $A_{2}>D_{2}$ and $\mathrm{B}_{2}>\mathrm{D}_{2}$ which share the same subtrahend. ${ }^{10}$

Figure 3 shows that there is indeed a very similar pattern of activation across the three subtractions. For all three contrasts, there are two main foci of preferential recruitment: BA 6 (left and bilateral) and visual cortex (primary/association). This said, type of subtraction also mattered: for the $A_{2}>D_{2}$ and $B_{2}>D_{2}$ contrasts, common preferential areas were revealed which did not emerge in the $\mathrm{B}_{2}>\mathrm{C}_{2}$ subtraction: anterior cingulate, $\mathrm{BA} 7$ (precuneus), and $\mathrm{BA} 32$. In addition, $\mathrm{B}_{2}>\mathrm{D}_{2}$ revealed activation of left BA 40. Finally, none of the contrasts showed overlap with BA 44 or BA 45-regions that were observed in the GAP-search condition. This finding was further confirmed in the double subtraction and conjunction analyses (see below). All results (from both Events 1 and 2) are summarized in Table 7 below.

The two GAP-completion-related double subtractions yielded interesting results. We predicted that if all Event 2 subtractions are targeting the same process, subtracting one from the other should result in no difference. And indeed that is what we found for $A_{2}>D_{2}$ vs. $B_{2}>D_{2}$. When these two conditions were compared to $\mathrm{B}_{2}>\mathrm{C}_{2}$ a difference was observed not in terms of localization but in terms of volume of activation. As Table 8 (see also Figure 4) shows, the activation pattern observed for these single and corresponding double subtractions is almost identical.

\footnotetext{
10 The activation in blue associated with the violation $C$ segment, $C_{2}$ vs. $B_{2}$, is addressed in the subsection "Other Patterns."
}

What we observe in the double-subtraction is a change in the volume for the SMA and which goes from 17,585 in the single $B_{2}>C_{2}$ subtraction down to 11,479 when subtracted by $A_{2}>D_{2}$ and to 13624 when subtracted by $\mathrm{B}_{2}>\mathrm{D}_{2}$. We compare these double subtractions to one where the GAP-search counterpart is subtracted: $\mathrm{B}_{2}>\mathrm{C}_{2}$ vs. $\mathrm{B}_{1}>\mathrm{C}_{1}$. We reason that if the previous two double subtractions are reflecting GAP-completion their results should converge with this one which isolates GAP-completion from GAP-search. And that is what we find. These results are summarized in Table 9. Finally, the conjunction analysis confirms these findings by showing again not only the primary and association visual cortex and connected posterior cortex, but crucially, BA 6 as a main area of overlap. These results are summarized in Table 10 and shown in Figure 5.

\subsection{Activation Beyond GAP-search and GAP-completion: Discourse-Composition and GAP Violation}

In the Event 2 contrast, an additional pattern of activation is observed which results from the inverse subtraction $\mathrm{C}_{2}>\mathrm{B}_{2}$ and which is associated with a GAP violation. (The violation is caused by an expected GAP that already appears filled.) This contrast was not part of the main question the study seeks to address, but in light of the other results, it reveals a very interesting pattern which we believe is connectable to our main question. The $\mathrm{C}_{2}>\mathrm{B}_{2}$ segment, which reflects the violation proper, recruits no LIF, LPST, or parietal cortices. Instead, it recruits the right hemisphere BAs 45 and 46 and bilateral prefrontal cortex (BAs 9 and 10).

This pattern is interesting because it reflects cortical recruitment beyond the traditional language areas, suggesting that its impact is outside language composition strictly speaking. Indeed in connection to this observation a reviewer points out, correctly in our view, that this pattern of activation lines up with the so-called default mode network (DMN); a network traditionally associated with resting states or situations where subjects are left to carry out "undirected" thinking. Consequently, the reviewer suggests, these could be an indication that the parser most likely has simply halted the comprehension process. 


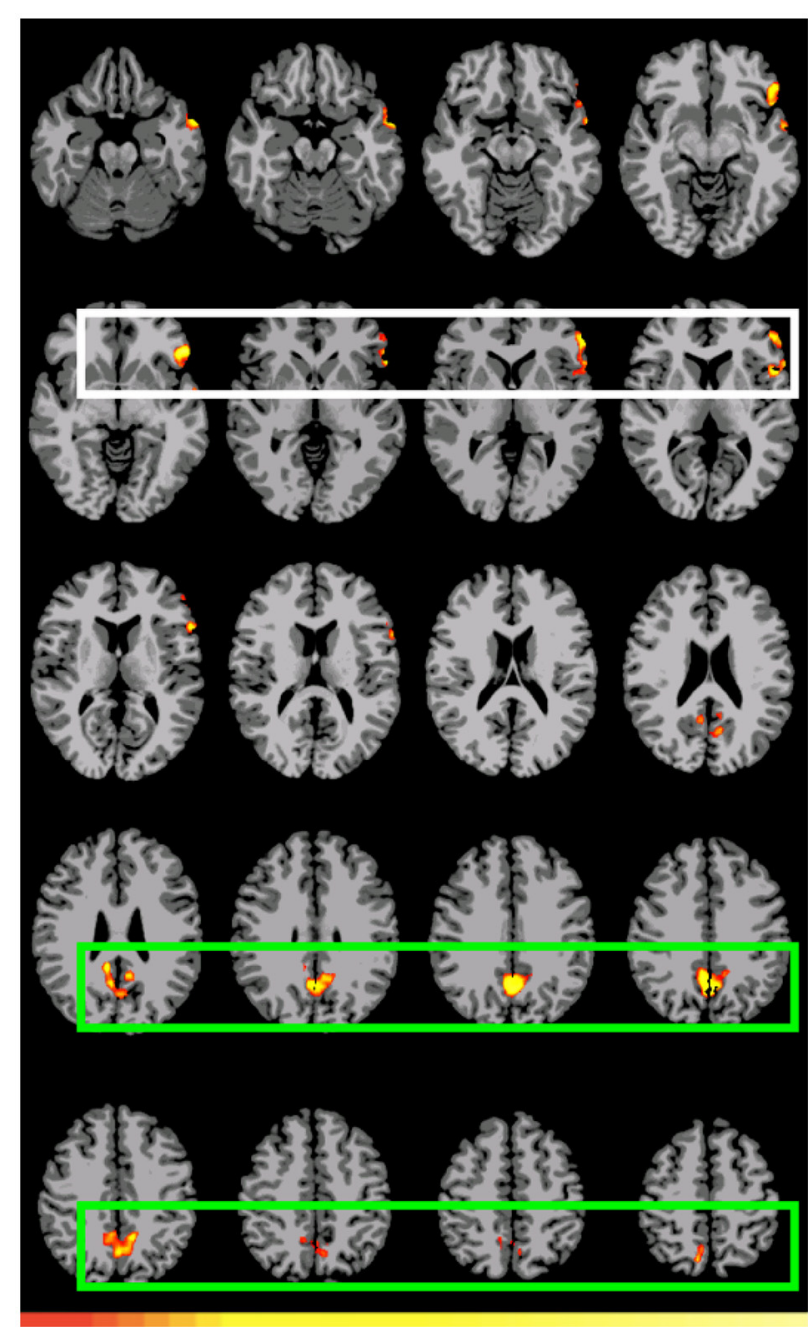

FIGURE 2 | Preferential activation for (direct) GAP-search (Event 1) double subtraction: $\left(\mathbf{A}_{\mathbf{1}}>\mathbf{D}_{\mathbf{1}}\right)$ vs. $\left(\mathbf{A}_{\mathbf{2}}>\mathbf{D}_{\mathbf{2}}\right)$. White: BAs $44,45,46$, and 47 . Green: Posterior cingulate and sensory association cortex. Images are shown corrected at $p<0.05$ in radiological format (LH is on the right). Only positive activation reported. Low thresholds $(p<0.05 ; t=2.14)$ are indicated by red, while high thresholds $(p<0.000005 ; t=7.20)$ are indicated by yellow.

We agree with the reviewer that to the extent that we do not fully know the impact of ungrammaticality in the process of comprehension, the possibility remains that faced with ungrammaticality, the comprehension system stops tracking linguistic composition altogether, thus allowing the mind to direct thought away from the utterance in question. This said, we would like to propose an alternative interpretation which is connectable with our present aims: that the pattern of preferential activation observed, partially overlapping with the default mode network, directly reflects the specific discourse-based nature of the violation in Condition C; a possibility that complements the recruitment pattern involved in gap-search/completion. On our analysis, the violation in Condition $\mathrm{C}$ is caused by the inability of the parser to integrate the composed meanings of the embedded and matrix clauses. These clauses are each independently syntactically and semantically well-formed yet cannot be linked with each other. The ill-formedness is caused by the requirement that GAP-completion apply at a point in the sentence where it is not allowed to. GAP-completion is the process where the referent associated with the antecedent finds an interpretation as a participant in the semantic representation associated with the embedded clause, thus linking the proposition denoted by the embedded clause with that of the matrix clause. In the ungrammatical utterance, The politician who the journalist's claim about the government report had bothered the people is calling a press conference, GAP-completion cannot take place because the GAP is already occupied by another NP (the people). Consequently, not only is the antecedent (the politician) left without a (necessary) interpretation within the embedded clause, but a new and unexpected semantic interpretation (involving the participant the people) has been introduced, which is locally plausible but cannot be connected with the meaning of the matrix clause. These two locally coherent segments (matrix clause: the politician is calling a press conference and embedded clause: the journalist's claim about the government report had bothered the people) result in compositionally conflicting linguistic representations, which in turn yield a meaning incoherence for the sentence as a whole (i.e., two mutually exclusive individuals "the politician" and "the people" must be licensed as the experiencer of "bother"). The meaning of the embedded clause (containing the new participant) can no longer be incorporated into the meaning of the matrix clause (containing the antecedent). This incoherence cannot be resolved not because there is no one plausible interpretation to be obtained, but because there is one too many plausible interpretations.

We propose that the comprehension system is sensitive to this situation and it is the conflict that it represents what underlies the activation pattern observed for $\mathrm{C}>\mathrm{B}$ event 2 . This would suggest in turn that the thrust of the violation lies on higher level meaning-based structure, even though the violation itself is triggered by a local syntactico-semantic misstep $^{11}$

If this were the case, it would make the non-linguistic regions in question relevant for language comprehension processes involving contextualization or integration of composed meaning. Early support for this possibility is found in fMRI reports suggesting a correlation of relevant right-hemisphere cortical areas with notions such as "discourse" level composition (e.g., Costello and Warrington, 1989; Devlin et al., 2003) and “aboutness" (Bornkessel-Schlesewsky et al., 2012). Specifically relevant to the DNM is the work on fMRI patterns relating the DMN to social cognition processes, in particular those connecting middle frontal cortex with theory of mind processes (see Mars et al., 2012 for a meta-analysis of this body of work in connection also to DMN processes in non-human primates). As noted, this interpretation is not intended to apply to syntactic violations across the board, but to activation

\footnotetext{
${ }^{11}$ This interpretation rests on a very specific assumption about the parser. The assumption is that the parser will attempt to build an interpretation even in the face of partial incoherence in the input as is the case in condition C. Yet as a reviewer correctly points out this assumption is not necessarily settled in the literature.
} 
TABLE 6 | Significant differential volumes by region for all GAP-completion (Event 2) contrasts.

\begin{tabular}{|c|c|c|c|c|c|}
\hline Contrast & Region & Vol. $\left(\mathrm{mm}^{3}\right)$ & Mean $T$-value & Max $T$-value & $\operatorname{Max} \operatorname{MNI}(x, y, z)$ \\
\hline \multirow[t]{3}{*}{$\mathrm{B}_{2}>\mathrm{C}_{2}$} & $\begin{array}{l}\text { Region 1: BA } 6 \text { (left, medial, bleeding into left BA } \\
\text { 44), left primary motor/sensory }\end{array}$ & 17,585 & 2.80 & 5.56 & $-39,-18, \quad 57$ \\
\hline & Region 2: visual cortex (primary/association) & 28,691 & 2.80 & 5.56 & $15,-87,-6$ \\
\hline & Region 3: caudate and putamen & 7820 & 2.80 & 5.56 & $18,21,6$ \\
\hline \multirow[t]{3}{*}{$A_{2}>D_{2}$} & $\begin{array}{l}\text { Region 1: BA } 6 \text { (bilateral, mostly medial/superior, } \\
\text { bleeding into BA 8), anterior and dorsal cingulate, } \\
\text { left primary motor/sensory }\end{array}$ & 19,052 & 2.98 & 7.61 & $-24, \quad-9, \quad 57$ \\
\hline & Region 2: BA 7, visual cortex (primary/association) & 45,564 & 3.13 & 8.56 & $-18,-69,-9$ \\
\hline & $\begin{array}{l}\text { Region 3: caudate, left putamen, left BA 47, left } \\
\text { insula }\end{array}$ & 5829 & 2.63 & 4.51 & $-30, \quad 21, \quad 0$ \\
\hline $\mathrm{B}_{2}>\mathrm{D}_{2}$ & Connected region: BA 6 (bilateral, medial, superior), & 72,530 & 2.97 & 9.07 & $-12,-87,-3$ \\
\hline
\end{tabular}

BA 8, anterior and dorsal anterior cingulate (right),

left primary motor/sensory, BA 7 (bleeding into BA

39), left BA 40, visual cortex (primary/association)
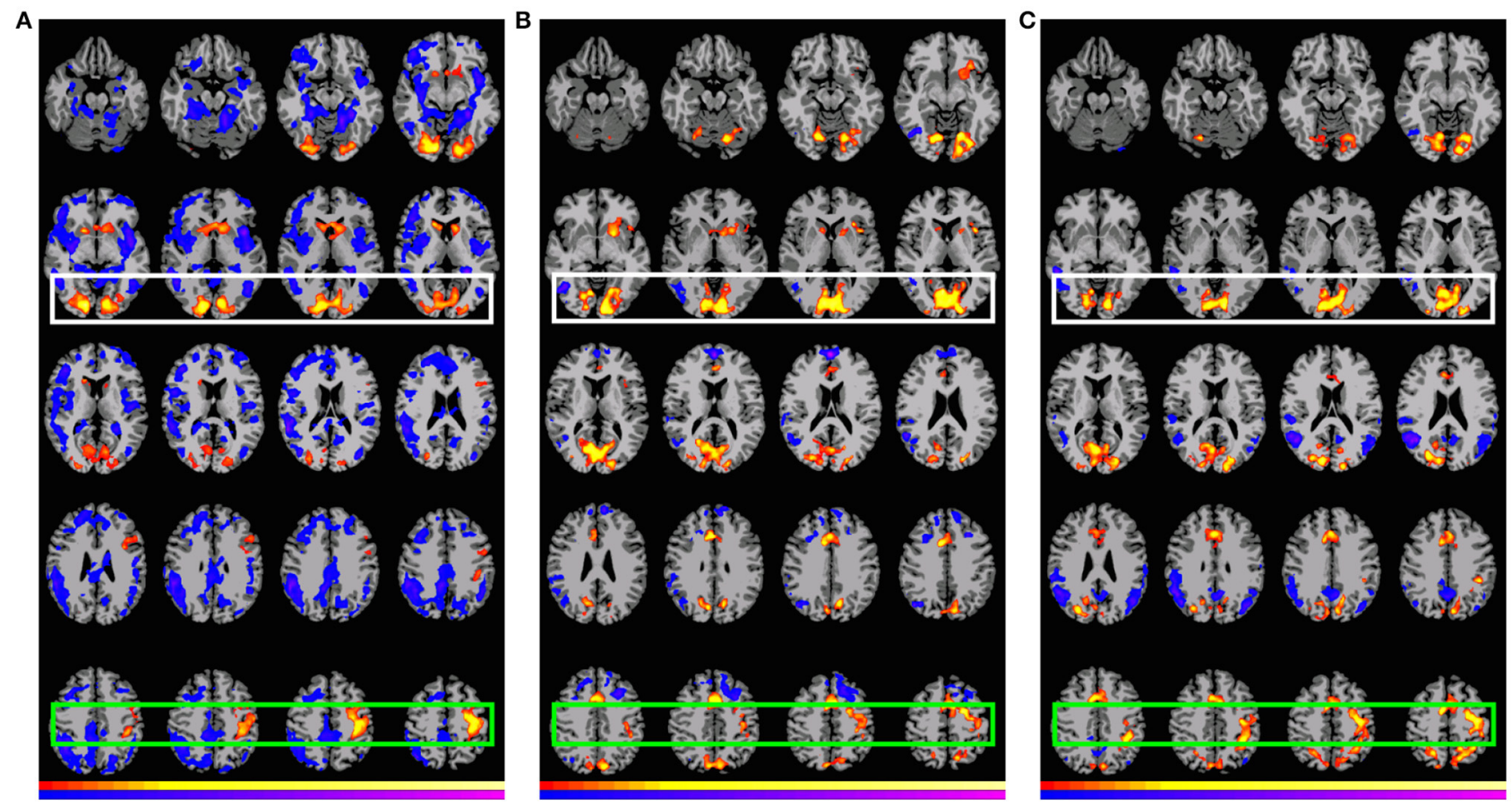

FIGURE 3 | Preferential (positive and negative) activation for GAP-completion (Event 2) subtractions. White: Visual cortex (association and primary), Green: SMA and parietal activation. Images are shown corrected at $p<0.05$ in radiological format (LH is on the right). For (A), low thresholds ( $p<0.05 ; t=2.14)$ are indicated by red and blue, while high thresholds $(p<0.000000005 ; t=12.6)$ are indicated by yellow and purple. For $(\mathbf{B})$, low thresholds $(p<0.05 ; t=2.14)$ are indicated by red and blue, while high thresholds $(p<0.0000006 ; t=8.6)$ are indicated by yellow and purple. For $(\mathbf{C})$, low thresholds $(p<0.05 ; t=2.14)$ are indicated by red (positive activation) and blue (negative activation), while high thresholds $(p<0.0000003 ; t=9.1)$ are indicated by yellow (positive activation) and purple (negative activation). (A) $B_{2}>C_{2}$ : "had bothered ${ }_{g a p}$ is calling a press conference." > "had bothered the people is calling a press conference." (B) $A_{2}>D_{2}$ : "had bothered gap is calling a press conference." > "had bothered the people and is calling a press conference." (C) $B_{2}>D_{2}$ : "had botheredgap is calling a press conference." > "had bothered the people and is calling a press conference."

patterns where the violation results in a larger discourse incoherence such as that created by a "doubly-filled" argument position. (For a more general discussion about brain patterns and violations, see Embick et al., 2000; Friederici et al., 2003).

\section{DISCUSSION}

Past neuroimaging work has shown that even though longdistance dependencies seem to recruit the workings of the LIF cortex, they also recruit the workings of the LPST cortex 
TABLE 7 | Summary of cortical recruitment by Events: Single subtractions.

\begin{tabular}{|c|c|c|}
\hline Subtraction & Event 1: GAP-search & Event 2: GAP-completion \\
\hline$B>C$ & - & $\begin{array}{l}\text { BA } 6 \text { (lateral), BA } 44 \text { (edge), visual cortex (BAs 17, 18, 19), caudate and putamen. } \\
\text { Activation is also observed in primary motor and primary sensory cortex. }\end{array}$ \\
\hline$A>D$ & $\begin{array}{l}\text { Region 1: left BA 45, left BA 44, left BA 47, insula, left } \\
\text { BA } 22 \text { (inferior, medial), BA } 38 \text { (temporal pole) Region } \\
\text { 2: posterior cingulate, left primary visual cortex, left } \\
\text { visual association cortex, bilateral BA } 7, \text { BA } 31\end{array}$ & $\begin{array}{l}\text { Region 1: BA } 6 \text { (bilateral, mostly medial/superior, bleeding into BA 8), anterior and dorsal } \\
\text { cingulate, left primary motor/sensory } \\
\text { Region 2: caudate/putamen (left), BA } 47 \text { (left), insula (left) } \\
\text { Region 3: BA } 7 \text { (bilateral), primary visual/association cortex (bilateral medial) }\end{array}$ \\
\hline$B>D$ & BA 6 (medial superior), BA 8, BA 24 (right), and BA 32. & $\begin{array}{l}\text { BA } 6 \text { (bilateral, medial, superior), BA 8, anterior and dorsal anterior cingulate (right), left } \\
\text { primary motor/sensory, BA } 7 \text { (bleeding into BA 39), left BA 40, visual cortex } \\
\text { (primary/association) }\end{array}$ \\
\hline
\end{tabular}

TABLE 8 | Significant differential volumes by region for all GAP-completion (Event 2) double subtractions.

\begin{tabular}{|c|c|c|c|c|c|}
\hline Contrast & Region & Vol. $\left(\mathrm{mm}^{3}\right)$ & Mean $T$-value & Max $T$-value & $\operatorname{Max} \operatorname{MNI}(x, y, z)$ \\
\hline \multirow[t]{2}{*}{$\left(B_{2}>C_{2}\right)$ vs. $\left(B_{2}>D_{2}\right)$} & Region 1: left BA 6, primary motor/sensory & 13264 & 3.11 & 7.18 & $-33,-18, \quad 60$ \\
\hline & Region 2: right visual cortex (primary/association) & 10579 & 3.67 & 12.07 & $15,-87,-6$ \\
\hline \multirow[t]{3}{*}{$\left(B_{2}>C_{2}\right)$ vs. $\left(B_{1}>C_{1}\right)$} & Region 1 left BA 6, primary motor/sensory, BA 8 & 18634 & 3.19 & 10.06 & $-39,-27, \quad 57$ \\
\hline & Region 2: visual cortex (primary/association) & 35814 & 3.42 & 12.05 & $15,-87,-6$ \\
\hline & Region 3: caudate, putamen, thalamus & 11848 & 2.68 & 5.12 & $18,21, \quad 12$ \\
\hline \multirow[t]{2}{*}{$\left(B_{2}>C_{2}\right)$ vs. $\left(A_{2}>D_{2}\right)$} & Region 1: left BA 6, primary motor/sensory & 11479 & 2.97 & 6.29 & $-51,-18, \quad 51$ \\
\hline & Region 2: right visual cortex (primary/association) & 9155 & 3.35 & 8.91 & $18,-84,-12$ \\
\hline
\end{tabular}

and surrounding areas (e.g., Cooke et al., 2002; Fiebach et al., 2002; Amunts et al., 2004; Fiebach et al., 2005; Grodzinsky and Friederici, 2006; Santi et al., 2015). Moreover, while the lexical role of the LPST cortex has been well documented (see Wise et al., 2001; Hickok and Poeppel, 2004, 2007 for proposals regarding the role of the various subcomponents of the LPST cortex in long-term phonological encoding), no conclusive explanation has been given for why this area should be recruited in the instantiation of these dependencies. At the same time, whereas Wernicke's patients (with damage involving the left posterior temporal cortex, including parts of the angular and supramarginal gyri) show across-the-board impaired sentence comprehension including constructions containing dependencies, they are indistinguishable from matching controls in their ability to exhibit the gap-filling effect, thus indicating that whatever their linguistic impairment, it does not seem to involve GAP-search or GAP-completion per se.

Indeed, Wernicke's performance has been seen to reflect the capacity to implement the basic syntactic mechanics of the dependency, but showing, offline, an inability to put this knowledge to use, presumably due to an inability to properly access the necessary lexico-semantic information that makes the dependency meaningful (e.g., Caramazza and Zurif, 1976; Shapiro and Levine, 1990; see Piñango and Zurif, 2015 for a summary of the main findings). By contrast, Broca's patients, while unable to properly implement these dependencies (e.g., Zurif et al., 1993, 1994; Burkhardt et al., 2003; Love et al., 2008), show, offline, a selective pattern of impairment whereby canonical (subject) relative clauses result in above-chance performance and non-canonical (object) relative clauses reliably result in poor (chance-level) comprehension, a pattern of performance that appears to be linguistic in nature. So whereas the neuroimaging evidence tells us the brain regions that could be potentially participating in the implementation of the dependencies, the lesion-based evidence tells us of the possibility of an asymmetry in their participation.

The analysis of LDDs that we present here provides the basis for a potential reconciliation of these two sets of seemingly conflicting observations by invoking organizing principles that could give rise to such an asymmetry. Specifically, the model captures the main linguistic components of a dependency (phrase structure building, argument structure licensing, and pronoun resolution) as selectional/subcategorization constraints on the relative pronoun that separate the process of searching for the environment of argument licensing within the sentence (GAP-search) from the actual argument licensing (GAP-completion).

In the remainder of this section we discuss the specific activation patterns observed in connection to the hypothesized functional distinctions.

\subsection{GAP-search: GAP-search direct $_{\text {vs. }}$ GAP-search indirect $_{\text {in }}$}

The hypothesis that LIF cortex is sensitive to GAP-search independently of the internal articulation of the dependency (direct vs. indirect) was not borne out. To the extent that GAP-search was reliably associated the LIF cortex it 

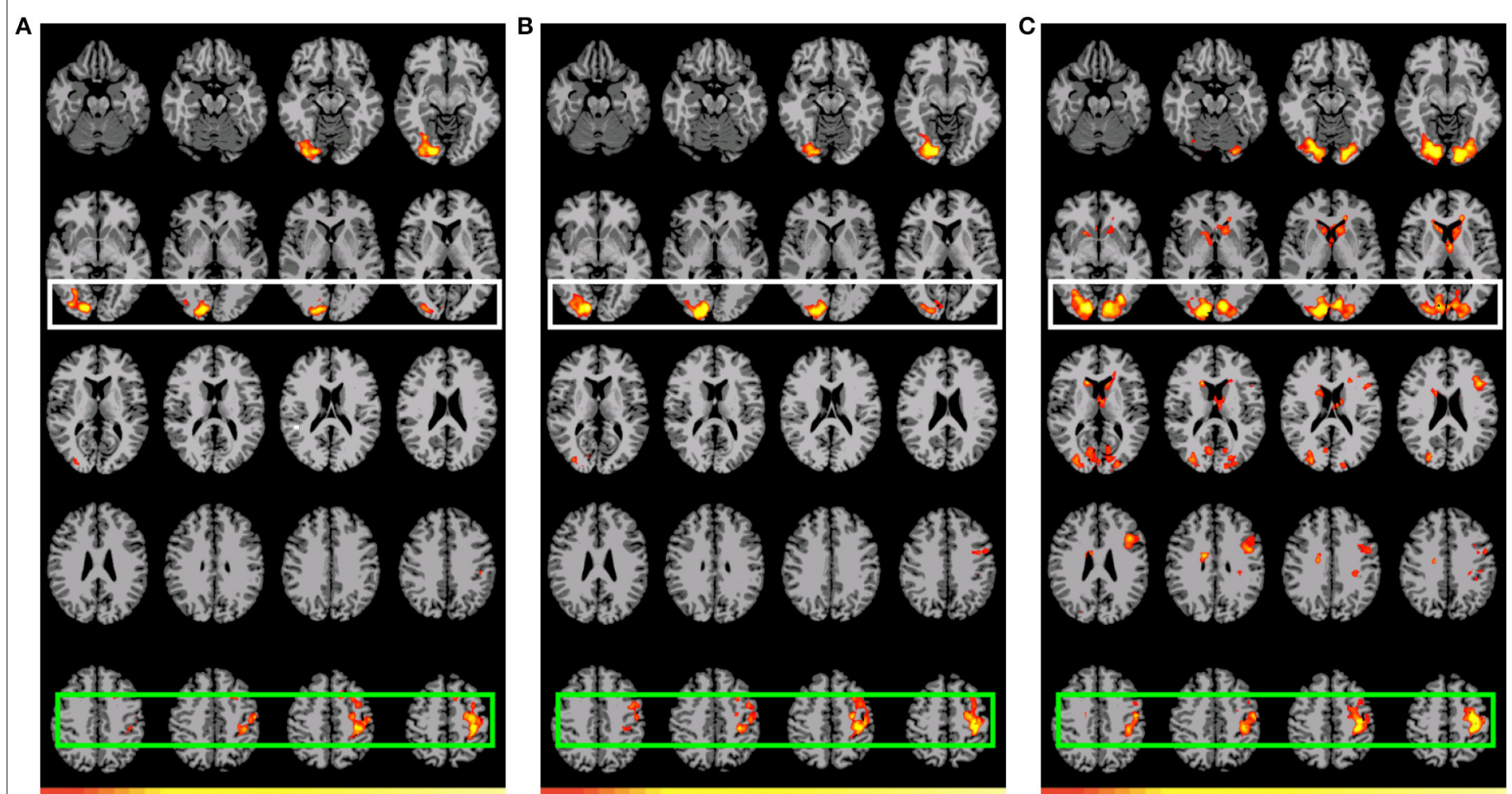

FIGURE 4 | Preferential positive activation for GAP-completion (Event 2) double subtractions. White: Visual cortex (association and primary), Green: SMA and parietal activation. Images are shown corrected at $p<0.05$ in radiological format ( $\mathrm{LH}$ is on the right). For (A), low thresholds $(p<0.05 ; t=2.14)$ are indicated by red, while high thresholds $(p<0.00000001 ; t=11.8)$ are indicated by yellow. For $(\mathbf{B})$, low thresholds $(p<0.05 ; t=2.14)$ are indicated by red, while high thresholds $(p<0.000000009 ; t=12.1)$ are indicated by yellow. For $(\mathbf{C})$, low thresholds $(p<0.05 ; t=2.14)$ are indicated by red, while high thresholds $(p<0.000000009 ; t=12.1)$ are indicated by yellow. (A) $\left(B_{2}>C_{2}\right)$ vs. $\left(A_{2}>D_{2}\right)$ : Residual preferential activation for $G A P$-completion. (B) $\left(B_{2}>C_{2}\right)$ vs. $\left(B_{2}>D_{2}\right)$ : Residual referential activation for GAP-completion. (C) $\left(B_{2}>C_{2}\right)$ vs. $\left(B_{1}>C_{1}\right)$ : Preferential activation for $G A P$-completion > preferential activation for GAP-search.

TABLE 9 | Summary of cortical recruitment for all GAP-completion (Event 2) double subtractions.

\begin{tabular}{|c|c|}
\hline Subtraction & Event 2: GAP-completion \\
\hline$\left(B_{2}>C_{2}\right)$ vs. $\left(A_{2}>D_{2}\right)$ & Region 1: left BA 6, primary motor/sensory cortex. Region 2: right primary/association visual cortex. \\
\hline$\left(B_{2}>C_{2}\right)$ vs. $\left(B_{2}>D_{2}\right)$ & Region 1: left BA 6, primary motor/sensory cortex. Region 2: right primary/association visual cortex. \\
\hline$\left(B_{2}>C_{2}\right)$ vs. $\left(B_{1}>C_{1}\right)$ & Region 1: left BA 6, primary motor/sensory cortex, BA 8. Region 2: primary/association visual cortex. Region 3: caudate, putamen, thalamus \\
\hline
\end{tabular}

TABLE 10 | Significant differential volumes by region for conjunction of GAP-completion (Event 2) subtractions, $\left(A_{2}>D_{2}\right)+\left(B_{2}>C_{2}\right)+\left(B_{2}>D_{2}\right)$.

\begin{tabular}{|c|c|c|c|c|}
\hline Region & Volume $\left(\mathrm{mm}^{3}\right)$ & Mean $T$-value & Max $T$-value & Max MNI Coords. (x, y, z) \\
\hline $\begin{array}{l}\text { Region } 1 \text { BA 6, motor (primary, supplementary), sensory (primary), } \\
\text { anterior cingulate }\end{array}$ & 5399 & $x$ & $x$ & $-2,-82, \quad 5$ \\
\hline $\begin{array}{l}\text { Region 2: right visual (association), right angular/supramarginal gyri, } \\
\text { fusiform }\end{array}$ & 4422 & $x$ & $x$ & $49,-55,19$ \\
\hline Region 3: BA7, BA19, visual (primary, association), sensory & 15,869 & $x$ & $x$ & $-33,-16,54$ \\
\hline
\end{tabular}

(association)

was only in connection to the direct condition (single and double subtractions). Within this pattern of activation two connected regions were involved: region 1 included BAs 45, 44, 47, bordering with the left insula and left temporal pole (anterior BAs 22 and 38). A second associated region connecting primary and associate visual cortex and BA7 and
BA31 were also preferentially recruited. This second region of activation is interesting for two reasons; (1) it appears in $A_{1}>D_{1}$ but not in the $B_{1}>D_{1}$ contrast, and this is relevant because it involves the participation of BA7, a cortical region previously connected to $\mathrm{CP}$ embedding, precisely the kind of composition present in $A_{1}$ and absent in $B_{1}$, and (2) it 
continues to appear in connection to GAP-completion for both $A_{2}>D_{2}$ and $B_{2}>D_{2}$ contrasts, thus suggesting that this area is sensitive to general composition such as that involved in gapcompletion $^{12}$.

The results from the $A_{1}>D_{1}$ vs. $A_{2}>D_{2}$ double subtraction, support the importance of the LIFG for GAP-search direct $_{\text {, }}$, an observation that replicates previous findings both from neuroimaging and lesion-studies. Those results further indicate that this cortical recruitment may at least be partly distinct from the cortical recruitment of GAP-completion.

Results also show that when GAP-search encounters a linguistic "obstacle"-as in Condition B (Event 1) GAPsearch $_{\text {indirect, }}$, and revealed in the $\mathrm{B}>\mathrm{D}$ (Event 1 ) contrast - a different preferential activation pattern emerges involving BA 6 (medial superior), BA 8, right BA 24, and BA 32. At the same time, results from the double-subtraction $A_{1}>D_{1}$ vs. $B_{1}>D_{1}$ reveal no preferential activation suggesting that these two conditions are also very similar. So, in light of the ambiguous statistical results, we offer an interpretation constrained by previous neuroimaging and lesion-based observations. We propose here that these two sets of results indicate there may not be a categorical distinction between the cortical regions engaged in GAP-search direct vs. those engaged in GAP-search indirect $_{\text {, }}$ instead the two reflect different patterns of activation within what is ultimately the same cortical network.

We thus interpret the LIF cortex preferential activation associated with GAP-search direct as resulting from an interaction of two factors involved in LDD resolution: (a) the prediction of a GAP, and (b) the possibility that the GAP be found within the syntactic and semantic contexts immediately after the RELPRO, that is, when nothing in the unfolding syntactic and semantic structure prevents the licensing of the GAP. These findings would thus represent independent neurological support for the existence of an active-filler (Clifton and Frazier, 1989; Frazier and Clifton, 1989; Fodor, 1995) that, crucially, is sensitive to the details of the linguistic context of the relative pronoun independently of the length of the dependency (Phillips et al., 2005).

Indeed, we take this pattern to reflect not necessarily a difference in search but a difference in quality of the search: when the parser is forced to use memory resources outside of the implementation of any specific linguistic mechanism -the delay caused by the parser's recognition that the expected GAP is not to be found in the current local constituent- those resources are recruited from cortical regions, most relevant BA 6 (SMA), which have been previously identified as participatory for language

\footnotetext{
${ }^{12} \mathrm{~A}$ reviewer asks us about our predictions for event 0 . We note that no $\mathrm{A} / \mathrm{B}>$ $\mathrm{D}$ difference was predicted and no difference was found at this segment. There are two reasons for this: (1) this early in the sentence, both A/B and D conditions show composition between the head noun and RELPRO, on the one case, and between the subject and the verb, on the other. Even though the nature of the composition that each carries is presumably different, we have no reason to expect that each will recruit visibly distinct cortical regions as a result. (2) Regarding the relative pronoun, even though it is true that by our definition GAP-search is triggered as soon as the RELPRO is retrieved, at this early point no structure has been built over which the search is to be carried out. So, even though GAP-search is triggered at event 0 , it will not be visible until the embedded clause is beginning to be built. This is precisely what the event 1 contrast is intended to reveal.
}

composition. The combined GAP-search pattern of results (direct plus indirect) would thus be reflecting the workings of two functional foci of the same linguistic network.

Support for this view is the observation that the LIF cortex and SMA have been traditionally connected, particularly in the focallesion literature (e.g., Benson, 1985; Tonkonogy, 1986; Vignolo, 1988; Naeser et al., 1989; Alexander et al., 1990; Goodglass, 1993).

This would mean in turn that the LIF cortex is sensitive to the expedient resolution of the dependency, which will only happen when such resolution is allowed by the local linguistic context. If it is not, then the preferential activation shifts (or reduces) to pre-SMA-all, however, within the same pathway.

This interpretation is consistent with Santi and Grodzinsky (2012) regarding the connection between "prediction" and the LIF cortex. Yet, what our results show is that presence of "prediction" is not enough. For the LIF cortex to be fully engaged, it must continuously be tracking for "gap-viability" as composition unfolds ${ }^{13}$.

Further elaborating on this issue, a reviewer suggests a perspective on the $B_{1}>D_{1}$ activation pattern that gives it a specific role-namely the suppression or inhibition of the direct GAP-search mechanism associated with the LIF cortex. In this view then, the monitoring action would presumably rely on the workings of the pre-SMA and in the situation where the GAPsearch could not take place, due to the island, it would act on the LIF cortex to suppress or hold search activity. We agree that this possibility, though outside the scope of the present data, is interesting and consistent with all other roles independently attributed to the SMA (e.g., Schwartze et al., 2012). Moreover, it brings the debate not only to a discussion of networks but to the possible distinguishable roles that their individual components may play during real-time cognitive processing.

Indeed, we take the activation of the supplementary motor area (SMA) in the $\mathrm{B}>\mathrm{D}$ (Event 1 ) contrast to be an important clue to the cortical recruitment of LDDs. Not only regarding GAP-search, but also GAP-completion as we will see below. Specifically, pre-SMA and SMA-proper (BA 6) have been independently shown to be involved in sensory-motor processing possibly manifested through a "gradient" in which sensory, nonsequential, suprasecond information is processed rostrally (recruiting pre-SMA cortex) while motoric, sequential, and subsecond information is processed more dorsally (Schwartze et al., 2012). Our present data are not fine-grained enough to reveal a dissociation between pre-SMA and SMA proper. However, the data do show the shared locus of activation to be on medial BA 6, suggesting the targeting of pre-SMA over SMAproper. Such a locus would be consistent with the processing of non-motoric, non-sequential, suprasecond information such as that involved in the holding of the filler in memory, as it were,

\footnotetext{
${ }^{13}$ These findings also connect directly to the cause of the abnormally delayed gap-filling observed in Broca's real-time comprehension (e.g., Burkhardt et al., 2003, Love et al., 2008). The combined behavior of $A>D$ and $B>D$ suggests that Broca's impairment may not be rooted to a generalized problem in syntactic structure formation (brought about in turn to a slowing in lexical retrieval), as has been proposed, but instead to the inability to engage the filler in an active manner as composition progresses, that is, to keep track of the viability of the syntactico-semantic structure being built.
} 


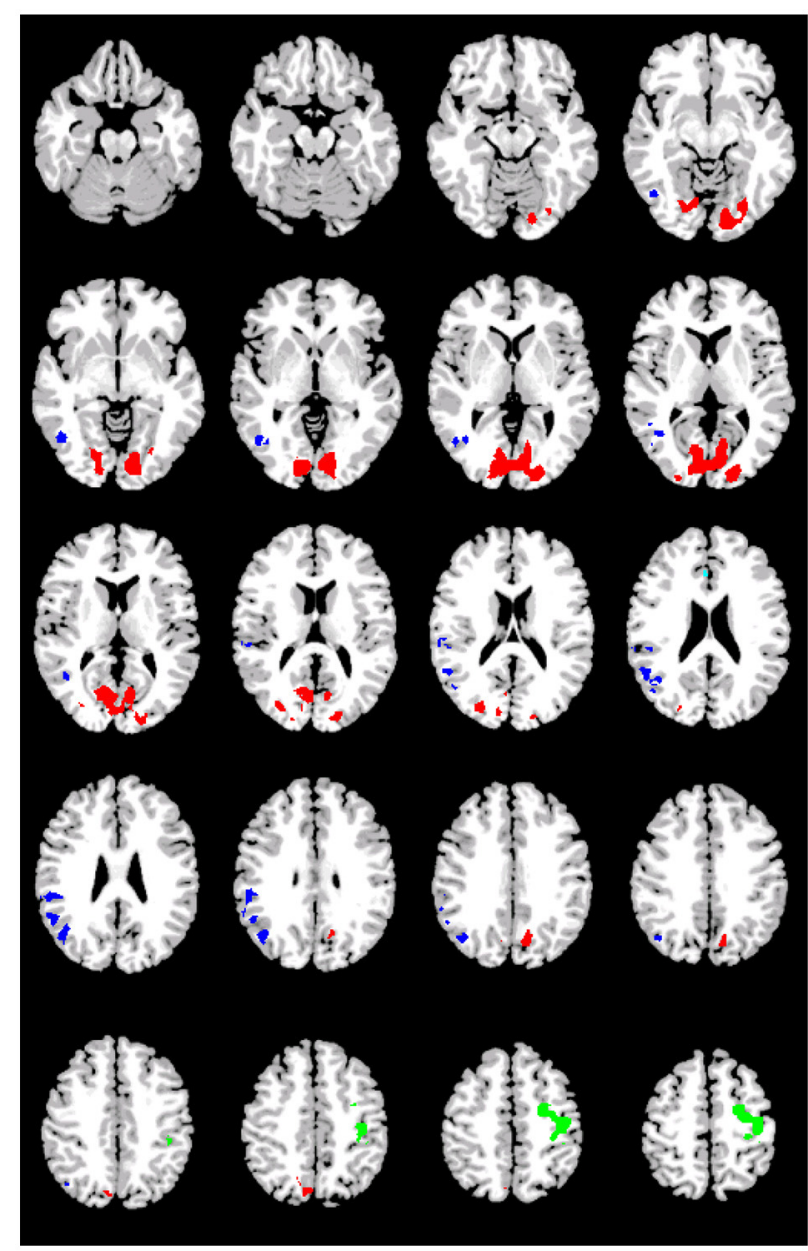

FIGURE 5 | Preferential activations for the conjunction of GAP-completion subtractions. Images are shown corrected at $p<0.05$ in radiological format ( $\mathrm{LH}$ is on the right). Green: BA6, primary motor and primary sensory connected with anterior cinculate (Acqua); Red: BA7, BA19, primary and association visual; Blue: right visual (association), right angular/ supramarginal gyri, and fusiform.

until the "GAP-unviable" segment has passed and active search can resume $e^{14}$.

\subsection{GAP-completion}

Regarding GAP-completion, our findings from the simple subtractions show that this mechanism recruits the workings of a contiguous cortical region within the left fronto-parietal lobes (and non-overlapping with those associated with (direct) GAP-search) connecting supplementary motor area, precuneus, and portions of the left angular and supramarginal gyri and peristriate (BA 19). This observation is further supported by all relevant double subtraction and conjunction analyses. What emerges then is a coherent language "network," as all of

\footnotetext{
${ }^{14}$ Interestingly, BA 6 and supplementary motor cortex have both been associated to Broca's aphasia and Transcortical Motor Aphasia, indicating a potential connection between the two syndromes, whose functional implications are still not well understood (Naeser et al., 1989; Alexander et al., 1990).
}

these areas have been independently connected with related components of language processing. Most critically, they have been associated with lexically-driven composition, such as that involving subcategorization (Shetreet et al., 2009) and lexicosemantic selectional restrictions (e.g., Lai et al., 2014). Indeed, we conjecture that this pattern of preferential activation is part and parcel of the "Dorsal Stream" or "Dorsal Pathway" (Hickok and Poeppel, 2004, 2007; Friederici, 2009, 2012), which connects the frontal and left posterior cortices via the parietal lobe. To the extent that this network is seen to be involved in a mechanism such as GAP-completion, a mechanism that brings together syntactic, lexico-semantic, and discourse composition, it tells us that this cortical region is at least partly recruited during unification of interpretation. And this would also be consistent with a version of the Memory, Unification and Control model (e.g., Hagoort, 2005, 2014) whereby the true locus of semantic unification includes, most crucially, at least the preSMA. It is in this way that the LPST cortex is connected to LDD implementation: as a potential participating region in a larger network that supports real-time lexically-driven language composition which, by definition, also supports GAP-completion.

One additional advantage of the connection between $G A P$ completion with the dorsal pathway is that it affords a possible explanation for the long-standing observation regarding Conduction aphasia comprehension first reported in Caramazza and Zurif (1976). Specifically, Caramazza and Zurif (1976) report that patients with Conduction aphasia (a syndrome associated with damage to the arcuate fasciculus) exhibit chance performance in the comprehension of semantically reversible (object) relative-clauses. Such a pattern is indistinguishable from that shown for Broca's comprehension but claimed to emerge from different causes. Caramazza and Zurif (1976) further note that, like Broca's, the pattern shown by Conduction patients contrasts sharply with that exhibited by Wernicke's patients, who show performance that is not attributable to any one linguistic or processing factor. Here we reason that if GAP-completion is dependent on the workings of the dorsal pathway, presumably connected to the arcuate fasciculus, it explains why Conduction patients would be impaired in the interpretation of semantically reversible relative clauses, despite being able to carry out GAP$\operatorname{search}^{15}$. In sum, we take the overall pattern accrued for all three Event 2, related double subtraction and conjunction contrasts to reflect components of this dorsal pathway, with BA 6 as a crucial area. This interpretation captures the normal-like performance by Wernicke's in online gap-filling constructions and suggests in turn that the LPST cortex activation from the imaging literature may not have been in connection to GAP-search proper.

In light of these findings, we are now able to address the questions posed in the introduction. What is the neurocognitive relation between GAP-search and GAP-completion? Answer: Their loci appears to be the LIF cortex and the (pre-)SMA, respectively. Do they rely on the workings of overlapping brain regions? Answer: The patterns we report show minimal overlap in recruitment. However, to the extent that at least the lower SMA

${ }^{15}$ The authors thank Julius Fridiksson (p.c) for reminding us of this long-standing yet unexplained observation. 
has been considered to be part of Broca's area, they are expected to functionally overlap. Our conjecture regarding the two areas [viable resolution (LIF cortex) vs. holding in memory (SMA)] is a proposal about how this overlap could take place. Can we associate GAP-completion to the LPST cortex, thus addressing the lesion-neuroimaging incongruence? Answer: we can if we understand Wernicke's area not as an isolated "language area" but as a part of a larger connectivity pathway "the dorsal stream" that connects Wernicke's area to the left fronto-parietal cortex including BA40, BA7 and the SMA. In line with the lesion-based literature, we conclude that LDD processing (defined in terms of $G A P$-search and GAP-completion) does not directly involve the preferential workings of Wernicke's area, but relies on areas that are functionally related to Wernicke's area.

Finally, if the effects reported reflect GAP-search, why are they observed mainly in the context of object-relative GAPs? Answer: what we find is that the effects reported reflect only an aspect of GAP-search, namely the requirement that the RELPRO be locally interpreted. But this only happens when GAP-search is being carried out over viable structure. So, it is as if the function of the LIF cortex is to monitor or keep track of the ability of the structure being composed to provide a GAP slot. In terms of our analysis, that amounts to keeping track of whether the selectional requirements of the RELPRO are being satisfied. As long as the composition signals that the GAP is incoming, the LIF cortex is fully engaged. From this perspective, then, the fact that this is observed mainly in object-GAP constructions is not a consequence of the grammatical feature per se, but of the fact that in these constructions, it takes longer for the RELPRO to be resolved as compared with subject-gap constructions, thus increasing the probability that the effect will be observed.

As a separate observation, our results also show that processing of memory-taxing sentential constructions (A and B) appear to systematically recruit the workings of the visual cortex (primary and association) areas (see Santi et al., 2015 and references therein for similar findings). We interpret this pattern separately for two reasons: (1) these areas are not traditionally associated with linguistic processing proper, and (2) this preferential activation was observed both during direct GAPsearch and GAP-completion, suggesting that the areas in question are not showing sensitivity to a specific linguistic process.

In light of this, we connect these findings to independent observations regarding the visual system and linguistic load, particularly in relation to pupillometry measures (see Piquado et al., 2010 for a review and additional experimental evidence in relation to language processing load and the visual system). That observation has been shown not to be restricted to cognitive effort, but to extend even to physical effort (Zénon et al., 2014). Accordingly, we take the visual cortex activation pattern to reflect the increased attention (i.e., effort) that the implementation of the relevant linguistic tasks represents but whose source may not be strictly linguistic (see (Martínez et al., 1999; Posner and Gilbert, 1999; Petersen and Posner, 2012) for observations specifically regarding non-visually related attention load and its impact on the visual cortex). In this respect we note that the visual cortex activation was not observed during indirect GAP-search further supporting the possibility that during the building of structure that is non-viable for a GAP, no search is actually taking place. And this would make this segment of comprehension less cognitively taxing.

\section{CONCLUSIONS: THE PRESENT RESULTS IN THE CONTEXT OF NEUROCOGNITIVE ARCHITECTURES}

In this section, we connect our results to larger neurocognitive architecture models. In this respect, we consider three models which address syntactic and/or semantic composition, the sort presumably directly involved in GAP-search and GAPcompletion. The first general observation is that whereas no one model accounts for the findings, each provides an insight into the larger pattern that the findings reflect. This gives us, then, the opportunity to focus on the common ground that each provides. This is what guides our discussion.

We start with Lau et al. (2008), who propose a model of semantic composition that could potentially involve LDD composition. In this model, the LIF cortex is connected to lexical retrieval. Interestingly our processing analysis of LDDs is lexically driven, and the key Event 1 contrast $A_{1}>D_{1}$ does vary the presence of the relative pronoun. However, as we have seen, lexical retrieval differences alone do not account for the activation pattern: specifically, the results from $B_{1}>D_{1}$, which also differ by the presence of the relative pronoun, do not show preferential LIF cortex activation. So, what is required in this model is a more precise treatment of the connection of lexical-retrieval to GAP-search in particular ${ }^{16}$.

The second model we consider is Friederici (2012), which proposes that language composition, understood as the process of building a semantic representation through syntactic structure, recruits the workings of the LIF cortex. To the extent that GAP-search has been isolated from syntactic structure building through the subtraction process, the model predicts the LIF cortex will not be involved in this process, a prediction that is not supported by the evidence. For the same reasons, the model does successfully predict the absence of activation of BAs 44 and 45 , particularly BA 44 , in $\mathrm{B}_{1}>\mathrm{D}_{1}$, which involves GAP-search but no hierarchical building. Friederici's (2012) model predicts no direct GAP-search in connection to the LIF cortex, because according to this model BAs 44 and 45 in particular are responsible for all syntactic structure building. Our results do not contradict this, but do point to the fact that BAs 44 and 45 must be additionally characterized as having specific compositional sensitivity, beyond generalized structure building. Finally, and as mentioned in the discussion, a most relevant aspect of Friederici's (2012) model (which also incorporates important insights from Hickok and Poeppel, 2004, 2007) involves the dorsal pathway, specifically,

\footnotetext{
${ }^{16}$ Relatedly, the more natural association with the workings of LDDs would be composition, since after all, the whole motivation for GAP-search is to compose the subject matrix nominal into the meaning of the embedded clause (and vice versa). But in the semantic model presented in Lau et al. (2008), this task is connected instead to the anterior temporal cortex (ATC) and angular gyrus, areas that are connectable instead to the ventral pathway and which, in our data, were only partly connected to GAP-search (in the form of the marginal activation of the temporal pole for $A_{1}>D_{1}$ ).
} 
Pathway I (also discussed in Friederici, 2009) and which connects the STG and BA 6 through the arcuate fasciculus. It is this pathway, we propose, that is responsible for compositional processes such as those represented by GAP-completion.

We reserve the end of this section for discussion of the Memory, Unification and Control (MUC) Model (e.g., Hagoort, 2005 , 2014). To our knowledge, this is the only model that explicitly assumes lexically-driven processing and grammatical systems, a feature that our processing analysis of LDDs also assumes. The model also capitalizes on the notion of unification, which provides a processing-friendly approach to composition. Like the Friederici (2012) model, Hagoort's 2005, 2014 model proposes a divide within the LIF cortex separating BAs 45 and 47 from BAs 44 and 45 for semantic and syntactic unification/processing functions, respectively. Whereas our data do not speak to the functional articulation within the LIF cortex, they do reveal that both subregions can at least work in tandem, as in the case of the activation for direct GAP-search. This is a reasonable interpretation, given that direct GAP-search involves both semantic and syntactic computations. What is not clear at this point is how unification should be understood such that it will include direct GAP-search as a mechanism while simultaneously excluding indirect GAP-search; both processes that are on the one hand "dynamic" in nature, and on the other highly sensitive to the linguistic context of the GAP. Another pending question is the nature of the connection between the LIF cortex and SMA/lower parietal cortex. Under MUC, these two regions could be involved in the same larger processing network, and the SMA activation observed could be part of the dynamics of the network triggered in turn by the linguistic properties of the sentence. In this interpretation, LDDs allow us to localize not two regions, but a network with two foci reflected in these two mechanisms. Since our data cannot speak directly to this point, this proposal remains to be supported.

To conclude, the results presented here suggest a resolution of the imaging vs. lesion incongruence by showing the privileging of BAs 45, 44, and 47 (over BA 6 and parietal and parietotemporal cortex, including the LPST cortex) in the process of direct GAP-search and by suggesting that the activation of LPST cortex reported in the neuroimaging literature is a manifestation of the workings of a network that supports other linguistic compositional processes associated instead with GAP-completion.

The results capture the inherent asymmetry between GAPsearch and GAP-completion and explain why damage to the LIF cortex would dramatically impact the ability of the comprehension system to complete the dependency, even if the cortical regions involved in GAP-completion remained intact. By the same token, to the extent that the evidence presented here does not involve the left posterior superior temporal cortex at

\section{REFERENCES}

Alexander, M. P., Naeser, M. A., and Palumbo, C. (1990). Broca's area aphasias: aphasia after lesions including the frontal operculum. Neurology 40, 353-362. doi: 10.1212/WNL.40.2.353

Amunts, K., Weiss, P. H., Mohlberg, H., Pieperhoff, P., Eickhoff, S., Gurd, J. M., et al. (2004). Analysis of neural mechanisms underlying verbal fluency in least directly, the results tell us why Wernicke's patients should not have issues in searching for and completing the GAP. Indeed, if our conjecture regarding the functional commitments of the SMA and the left lower parietal region (associated with GAPcompletion) to compositional unification is correct, Wernicke's patients, who have been shown to have lexical retrieval problems, should not show problems in finding/completing the GAP but in unifying this information with the matrix clause into an interpretable string. Such a situation would lead to across-the board comprehension problems in these patients, a prediction that evidence from offline comprehension of these patients (in contrast to Broca's patients) consistently supports.

\section{AUTHOR CONTRIBUTIONS}

MP is responsible for the conception of the work and participated in each aspect of the project including experimental design, data acquisition and analysis, and drafting of the work. EF is responsible for stimuli generation and norming, subject recruitment, data acquisition, data analysis planning (e.g., timing file generation), interpretation and drafting of the work, and participated in the final approval of the version to be published. CL carried out all the data analysis and participated in the final approval of the version to be published. RC participated in the experimental design, data analysis and interpretation, drafting of the work, and in the final approval of the version to be published.

\section{FUNDING}

This project was funded by NSF BCS-0643266 awarded to MP and NSF-INSPIRE 1248100 awarded to MP, Ashwini Deo, Mokshay Madiman, and RC.

\section{ACKNOWLEDGMENTS}

We thank Jeetu Bhawnani and Hedy Sarofin for programming and technical support through the data acquisition stage. We also thank Emily Foster Hanson, Sara Sanchez-Alonso, and Muye Zhang for key assistance with aspects of the data analysis and data presentation. Finally, we are grateful to Edgar Zurif for much discussion on the gap-filling effect and the neurocognition of language composition; discussion that has directly impacted the approach taken here. All errors remain our own.

\section{SUPPLEMENTARY MATERIAL}

The Supplementary Material for this article can be found online at: http://journal.frontiersin.org/article/10.3389/fpsyg. 2016.01434

cytoarchitectonically defined stereotaxic space: the roles of Brodmann areas 44 and 45. Neuroimage 22, 42-56. doi: 10.1016/j.neuroimage.2003.12.031

Avrutin, S. (1999). Development of the Syntax-Discourse Interface, Vol. 23. Norwell: Kluwer; Springer Science and Business Media. doi: 10.1007/978-94-017-1239-2 Avrutin, S. (2006). "Weak syntax," in Broca's Region, eds Y. Grodzinsky and L. Amunts (Norwell: Oxford University Press), 179-183. doi: 10.1093/acprof:oso/ 9780195177640.003 .0004 
Ben-Shachar, M., Hendler, T., Kahn, I., Ben-Bashat, D., and Grodzinsky, Y. (2003). The neural reality of syntactic transformations: evidence from functional magnetic resonance imaging. Psychol. Sci. 14, 433-440. doi: 10.1093/acprof:oso/9780195177640.003.0004

Ben-Shachar, M., Palti, D., and Grodzinsky, Y. (2004). Neural correlates of syntactic movement: converging evidence from two fMRI experiments. NeuroImage 21, 1320-1336. doi: 10.1016/j.neuroimage.2003.11.027

Benson, F. (1985). "Aphasia," in Clinical Neuropsychology, eds K. Heilman and E. Valenstein (New York, NY: Oxford University Press), 17-48.

Bornkessel-Schlesewsky, I., Grewe, T., Schlesewsky, M. (2012). Prominence vs. aboutness in sequencing: a functional distinction within the left inferior frontal gyrus. Brain Lang. 120, 96-107. doi: 10.1016/j.bandl.2010.06.004

Bresnan, J. (1982). The Mental Representation of Grammatical Relations, Vol. 1. Cambridge, MA: The MIT Press.

Bresnan, J. (2001). "The emergence of the unmarked pronoun," in OptimalityTheoretic Syntax, eds G. Legendre, S. Vikner, and J. Grimshaw (Oxford: Blackwell Publishers Ltd.), 45-50.

Burkhardt, P., Piñango, M. M., and Wong, K. (2003). The role of the anterior left hemisphere in real-time sentence comprehension: evidence from split intransitivity. Brain Lang. 86, 9-22. doi: 10.1016/S0093-934X(02) 00526-6

Caramazza, A., and Zurif, E. (1976). Dissociation of algorithmic and heuristic processes in language comprehension: evidence from aphasia. Brain Lang. 3, 572-582. doi: 10.1016/0093-934X(76)90048-1

Chomsky, N. (1965). Aspects of a Theory of Syntax. Cambridge, MA: The MIT Press.

Chomsky, N. (1981). "Principles and parameters in syntactic theory," in Explanation in Linguistics: The Logical Problem of Language Acquisition, Vol. 32, Lectures on Government and Binding: The Pisa Lectures (Holland: Foris Publications), 75.

Clifton, C. Jr., and Frazier, L. (1989). "Comprehending sentences with longdistance dependencies" in Linguistic Structure in Language Processing, eds G. Carlson and M. Tanenhaus (Dordrecht: Kluwer), 273-317. doi: 10.1007/97894-009-2729-2_8

Crain, S., and Fodor, J. D. (1985). "How can grammars help parsers?," in Natural Language Parsing: Psychological, Computational Ad Theoretical Perspectives, eds D. Dowty, L. Karttunen, and A. Zwicky (Cambridge: Cambridge University Press), 24-128.

Cooke, A., Zurif, E. B., DeVita, C., Alsop, D., Koenig, P., Detre, J., et al. (2002). Neural basis for sentence comprehension: grammatical and shortterm memory components. Hum. Brain Mapp. 15, 80-94. doi: 10.1002/hbm. 10006

Costello, A. L., and Warrington, E. K. (1989). Dynamic aphasia: the selective impairment of verbal planning. Cortex 25, 103-114. doi: 10.1016/S00109452(89)80010-3

Culicover, P. W., and Jackendoff, R. (2005). Simpler Syntax. Oxford: Oxford University Press. doi: 10.1093/acprof:oso/9780199271092.001.0001

Devlin, J. T., Matthews, P. M., and Rushworth, M. F. (2003). Semantic processing in the left inferior prefrontal cortex: a combined functional magnetic resonance imaging and transcranial magnetic stimulation study. J. Cogn. Neurosci. 15, 71-84. doi: 10.1162/089892903321107837

Embick, D., Marantz, A., Miyashita, Y., O’Neil, W., and Sakai, K. L. (2000). A syntactic specialization for Broca's area. Proc. Natl. Acad. Sci. U.S.A. 97, 6150-6154. doi: 10.1073/pnas.100098897

Evans, A. C., Marrett, S., Neelin, P., Collins, L., Worsley, K., Dai, W., et al. (1992). Anatomical mapping of functional activation in stereotactic coordinate space. Neuroimage 1, 43-53. doi: 10.1016/1053-8119(92)90006-9

Fiebach, C., Schlesewsky, M., and Friederici, A. (2002). Separating syntactic memory costs and syntactic integration costs during parsing: the processing of German wh-questions. J. Mem. Lang. 47, 250-272. doi: 10.1016/S0749596X(02)00004-9

Fiebach, C., Schlewesky, M., Lohmann, G., von Cramon, D., and Friederici, A. (2005). Revisiting the role of Broca's area in sentence processing: syntactic integration versus syntactic working memory. Hum. Brain Mapp. 24, 79-91. doi: $10.1002 / \mathrm{hbm} .20070$

Fillmore, C. J. (1988). "The mechanisms of "construction grammar," in Annual Meeting of the Berkeley Linguistics Society, Vol. 14 (Berkeley, CA), 35-55. doi: 10.3765/bls.v14i0.1794
Fodor, J. (1995). "Comprehending sentence structure," in An Invitation to Cognitive Science: Language, eds D. Osherson, L. Gleitman, and M. Liberman (Cambridge, MA: The MIT Press), 209-246.

Frazier, L., Clifton, C., and Randall, J. (1983). Filling gaps: decision principles and structure in sentence comprehension. Cognition 13, 187-222. doi: 10.1016/0010-0277(83)90022-7

Frazier, L., and Clifton, C. (1989). Successive cyclicity in the grammar and the parser. Lang. Cogn. Process. 4, 93-126. doi: 10.1080/01690968908406359

Frazier, L., and Flores d'Arcais, G. (1989). Filler-driven parsing: a study of gap filling in Dutch. J. Mem. Lang. 28, 331-344.

Friederici, A. D., Rüschemeyer, S. A., Hahne, A., and Fiebach, C. J. (2003). The role of left inferior frontal and superior temporal cortex in sentence comprehension: localizing syntactic and semantic processes. Cereb. Cortex 13, 170-177. doi: 10.1093/cercor/13.2.170

Friederici, A. D. (2009). Pathways to language: fiber tracts in the human brain. Trends Cogn. Sci. 13, 175-181. doi: 10.1016/j.tics.2009. 01.001

Friederici, A. D. (2012). The cortical language circuit: from auditory perception to sentence comprehension. Trends Cogn. Sci. 16, 262-268. doi: 10.1016/j.tics.2012.04.001

Gibson, E. (1998). Linguistic complexity: locality of syntactic dependencies. Cognition 68, 1-76. doi: 10.1016/S0010-0277(98)00034-1

Gibson, E., and Warren, T. (2004). Reading-time evidence for intermediate linguistic structure in long-distance dependencies. Syntax 7, 55-78. doi: $10.1111 / j .1368-0005.2004 .00065 . x$

Goldberg, A. E. (1995). Constructions: A Construction Grammar Approach to Argument Structure. Chicago: University of Chicago Press.

Goodglass, H. (1993). Understanding Aphasia. San Diego, CA: Academic Press.

Grodzinsky, Y. (1989). Agrammatic comprehension of relative clauses. Brain Lang. 37, 480-499. doi: 10.1016/0093-934X(89)90031-X

Grodzinsky, Y., Pierce, A., and Marakovitz, S. (1991). Neuropsychological reasons for a transformational analysis of verbal passive. Nat. Lang. Linguist. Theory 9 , 431-453. doi: 10.1007/BF00135354

Grodzinsky, Y., Wexler, K., Chien, Y. C., Marakovitz, S., and Solomon, J. (1993). The breakdown of binding relations. Brain Lang. 45, 396-422. doi: 10.1006/brln.1993.1052

Grodzinsky, Y., Piñango, M. M., Zurif, E., and Drai, D. (1999). The critical role of group studies in neuropsychology: comprehension regularities in Broca’s aphasia. Brain Lang. 67, 134-147. doi: 10.1006/brln.1999. 2050

Grodzinsky, Y. (2000). The neurology of syntax: language use without Broca's area. Behav. Brain Sci. 23, 1-21. doi: 10.1017/S0140525X00002399

Grodzinsky, Y., and Friederici, A. D. (2006). Neuroimaging of syntax and syntactic processing. Curr. Opin. Neurobiol. 16, 240-246. doi: 10.1016/j.conb.2006.03.007

Hagoort, P. (2005). On Broca, brain, and binding: a new framework. Trends Cogn. Sci. 9, 416-423. doi: 10.1016/j.tics.2005.07.004

Hagoort, P. (2014). Nodes and networks in the neural architecture for language: Broca's region and beyond. Curr. Opin. Neurobiol. 28, 136-141. doi: 10.1016/j.conb.2014.07.013

Hickok, G. (1993). Parallel parsing: evidence from reactivation in garden-path sentences. J. Psycholinguist. Res. 22, 239-250.

Hickok, G., and Poeppel, D. (2004). Dorsal and ventral streams: a framework for understanding aspects of the functional anatomy of language. Cognition 92, 67-99. doi: 10.1016/j.cognition.2003.10.011

Hickok, G., and Poeppel, D. (2007). The cortical organization of speech processing. Nat. Rev. Neurosci. 8, 393-402. doi: 10.1038/nrn2113

Holmes, C. J., Hoge, R., Collins, L., Woods, R., Toga, A. W., and Evans, A. C. (1998). Enhancement of MR images using registration for signal averaging. J. Comput. Assist. Tomogr. 22, 324-333. doi: 10.1097/00004728-19980300000032

Husband, E. M., Kelly, L. A., and Zhu, D. C. (2011). Using complement coercion to understand the neural basis of semantic composition: evidence from an fMRI study. J. Cogn. Neurosci. 23, 3254-3266. doi: 10.1162/jocn_a_ 00040

King, J., and Kutas, M. (1995). Who did what and when? Using word- and clause level ERPs to monitor working memory usage in reading. J. Cogn. Neurosci. 7, 376-395. doi: 10.1162/jocn.1995.7.3.376 
Kluender, R. (1998). "On the distinction between strong and weak islands: a processing perspective," in Syntax and Semantics 29: The Limits of Syntax, eds P. Culicover and L. McNally (San Diego, CA: Academic Press), 241-279.

Lai, Y.-Y., Lacadie, C., Constable, T., Deo, A., and Piñango, M. M. (2014). "Complement coercion as the processing of aspectual verbs: evidence from self-paced reading and fMRI," in Proceedings of the 36th Annual Conference of the Cognitive Science Society, eds P. Bello, M. S. Guarini, M. McShane, and B. Scassellati (Austin, TX: Cognitive Science Society), 2525-2530.

Lai, Y.-Y., Lacadie, C., Constable, T., Deo, A., and Piñango, M. M. (in press). "Complement coercion as the processing of aspectual verbs: evidence from selfpaced reading and fMRI," in Compositionality and Concepts in Linguistics and Psychology, eds Y. Winter and J. Hampton (London: Springer).

Lau, E. F., Phillips, C., and Poeppel, D. (2008). A cortical network for semantics: (de)constructing the N400. Nat. Rev. Neurosci. 9, 920-933. doi: $10.1038 / \mathrm{nrn} 2532$

Love, T., Swinney, D., Walenski, M., and Zurif, E. (2008). How left inferior frontal cortex participates in syntactic processing: evidence from aphasia. Brain Lang. 107, 203-219. doi: 10.1016/j.bandl.2007.11.004

Martínez, A., Anllo-Vento, L., Sereno, M. I., Frank, L. R., Buxton, R. B., Dubowitz, D. J., et al. (1999). Involvement of striate and extrastriate visual cortical areas in spatial attention. Nat. Neurosci. 2, 364-369. doi: 10.1038/ 7274

Makuuchi, M., Bahlmann, J., Anwander, A., and Friederici, A. D. (2009). Segregating the core computational faculty of human language from working memory. Proc. Natl. Acad. Sci. U.S.A. 106, 8362-8367. doi: 10.1073/pnas.0810928106

Mars, R. B., Neubert, F., Noonan, M. P., Sallet, J., Toni, I., and Rushworth, M. F. S. (2012). On the relationship between the "default mode network" and the "social brain.” Front. Hum. Neurosci. 6:189. doi: 10.3389/fnhum.2012.00189

MacDonald, M. C. (1989). Priming effects from gaps to antecedents. Lang. Cogn. Process. 4, 1-72. doi: 10.1080/01690968908406356

McElree, B., and Bever, T., (1989) The psychological reality of linguistically defined gaps. J. Psycholinguist. Res. 18, 21-35. doi: 10.1007/BF01069044

Matchin, W., Sprouse, J., and Hickok, G. (2014). A structural distance effect for backward anaphora in Broca's area: an fMRI study. Brain Lang. 138, 1-11. doi: 10.1016/j.bandl.2014.09.001

Naeser, M. A., Palumbo, C. D., Helm-Estabrooks, N., Stiassny-Eder, D., and Albert, M. L. (1989). Severe nonfluency in aphasia. Brain 112, 1-38. doi: 10.1093/brain/112.1.1

Nicol, J., and Swinney, D. (1989). The role of structure in coreference assignment during sentence comprehension. J. Psycholinguist. Res. 18, 5-19. doi: 10.1007/BF01069043

Nolfe, E. X., Voet, T., Jacobs, F., Dierckx, R., and Lemahieu, I. X. (2003). An open-source medical image conversion toolkit. Eur. J. Nucl. Med. 30(Suppl. 2):S246.

Papademetris, X., Jackowski, M. P., Rajeevan, N., DiStasio, M., Okuda, H., Constable, R. T., et al. (2006). BioImage suite: an integrated medical image analysis suite: an update. Insight J. 2006:209.

Petersen, S. E., and Posner, M. I. (2012). The attention system of the human brain: 20 years after. Ann. Rev. Neurosci. 35, 73-89. doi: 10.1146/annurev-neuro062111-150525

Phillips, C. (2003). Linear order and constituency. Linguist. Inq. 34, 37-90. doi: $10.1162 / 002438903763255922$

Phillips, C., Kazanina, N., and Abada, S. (2005). ERP effects of the processing of syntactic long-distance dependencies. Cogn. Brain Res. 22, 407-428. doi: 10.1016/j.cogbrainres.2004.09.012

Piñango, M. M., and Zurif, E. B. (2001). Semantic operations in aphasic comprehension: implications for the cortical organization of language. Brain Lang. 79, 297-308. doi: 10.1006/brln.2001.2492

Pinango, M. M. (2003). "Cortical reflections of two pronominal relations," in Pronouns: Grammar and Representations, eds H. Simon and H. Wiese (Berlin: Verlag), 232-252.

Piñango, M. M., and Burkhardt, P. (2005). "Pronominal interpretation and the syntax-discourse interface: real-time comprehension and neurological properties," in Anaphora Processing: Linguistic, Cognitive and Computational Models, eds A. Branco, T. McEnery, and
R. Mitkoy (Amsterdam: John Benjamins Publishing Company), 221-238.

Piñango, M. M., and Zurif, E. (2015). "An evolving view of enriched semantic composition," in Conceptual Semantics, eds P. Csuri and I. Toivonen (Cambridge, MA: MIT Press), 167-186.

Piquado, T., Isaacowitz, D., and Wingfield, A. (2010). Pupillometry as a measure of cognitive effort in younger and older adults. Psychophysiology 47, 560-569. doi: 10.1111/j.1469-8986.2009.00947.x

Posner, M. I., and Gilbert, C. D. (1999). Attention and primary visual cortex. Proc. Natl. Acad. Sci. U.S.A. 96, 2585-2587. doi: 10.1073/pnas.96.6. 2585

Santi, A., and Grodzinsky, Y. (2007). Working memory and syntax interact in Broca's area. NeuroImage 37, 8-17. doi: 10.1016/j.neuroimage.2007. 04.047

Santi, A., and Grodzinsky, Y. (2008). The battle for Broca's region. Trends Cogn. Sci. 12, 474-480. doi: 10.1016/j.tics.2008.09.001

Santi, A., and Grodzinsky, Y. (2010). fMRI adaptation dissociates syntactic complexity dimensions NeuroImage 51, 1285-1293. doi: 10.1016/j.neuroimage.2010.03.034

Santi, A., and Grodzinsky, Y. (2012). Broca's area and sentence comprehension: a relationship parasitic on dependency, displacement or predictability? Neuropsychologia 50, 821-832. doi: 10.1016/j.neuropsychologia.2012. 01.017

Santi, A., Friederici, A. D., Makuuchi, M., and Grodzinsky, Y. (2015). An fMRI study dissociating distance measures computed by Broca's area in movement processing: clause boundary vs identity. Front. Psychol. 6:654. doi: 10.3389/fpsyg.2015.00654

Schumacher, P. B., Piñango, M. M., Ruigendijk, E., and Avrutin, S. (2010). Reference assignment in Dutch: evidence for the syntaxdiscourse divide. Lingua 120, 1738-1763. doi: 10.1016/j.lingua.2009. 12.003

Schwartze, M., Rothermich, K., and Kotz, S. (2012). Functional dissociation of preSMA and SMA-proper in temporal processing. NeuroImage 60, 290-298. doi: 10.1016/j.neuroimage.2011.11.089

Shapiro, L. P., and Levine, B. A. (1990). Verb processing during sentence comprehension in aphasia. Brain Lang. 38, 21-47. doi: 10.1016/0093934X(90)90100-U

Shetreet, E., Friedmann, N., and Hadar, U. (2009). An fMRI study of syntactic layers: sentential and lexical aspects of embedding. Neuroimage 48, 707-716. doi: 10.1016/j.neuroimage.2009.07.001

Stowe, L. (1986). Evidence for on-line gap location. Lang. Cogn. Process. 1, 227-245. doi: 10.1080/01690968608407062

Stromswold, K., Caplan, D., Alpert N., and Rauch, S. (1996). Localization of syntactic comprehension by positron emission tomography. Brain Lang. 52, 452-473. doi: 10.1006/brln.1996.0024

Swinney, D., Ford, M., Frauenfelder, U., and Bresnan, J. (1988). "On the temporal course of gap-filling and antecedent assignment during sentence comprehension," in Language Structure and Processing, eds B. Grosz, R. Kaplan, M. Macken, and I. Sag (Standord, CA: Center for the Study of Language and Information).

Swinney, D., Zurif, E. B., and Nicol, J. (1989). The effects of focal brain damage on sentence processing: an examination of the neurological organization of a mental module. J. Cogn. Neurosci. 1, 25-37. doi: 10.1162/jocn.1989. 1.1 .25

Swinney, D., and Zurif, E. (1995). Syntactic processing in aphasia. Brain Lang. 50, 225-239. doi: 10.1006/brln.1995.1046

Swinney, D., Zurif, E., Prather, P., and Love, T. (1996). Neurological distribution of processing resources underlying language comprehension. J. Cogn. Neurosci. 8, 174-184. doi: 10.1162/jocn.1996.8.2.174

Tonkonogy, J. (1986). Vascular Aphasia. Cambridge, MA: MIT Press.

Van Valin, R. D., and LaPolla, R. J. (1997). Syntax: Structure, Meaning, and Function. Cambridge: Cambridge University Press. doi: 10.1017/cbo97811391 66799

Vignolo, L. (1988). "The anatomical and pathological basis of aphasia," in Aphasia, eds E. C. Rose, R. Whurr, and M. A. Wyke (London: Whurr), 227-249.

Wise, R., Scott, S., Blank, S., Mummery, C., Murphy, K., and Warburton, E. (2001). Separate neural systems within Wernicke's area. Brain 142, 83-95. doi: 10.1093/brain/124.1.83 
Zénon, A., Sidibé, M., and Olivier, E. (2014). Pupil size variations correlate with physical effort perception. Front. Behav. Neurosci. 8:286. doi: 10.3389/fnbeh.2014.00286

Zurif, E. B., Swinney, D., Prather, P., Solomon, J., and Bushell, C. (1993). An on-line analysis of syntactic processing in Broca's and Wernicke's aphasia. Brain Lang. 45, 448-463. doi: 10.1006/brln. 1993.1054

Zurif, E., Swinney, D., Prather, P., and Love, T. (1994). Functional localization in the brain with respect to syntactic processing. J. Psycholinguist. Res. 23, 487-498. doi: 10.1007/BF02146687

Zurif, E., Swinney, D., Prather, P, Wingfield, A., and Brownell, H. (1995). The allocation of memory resources during sentence comprehension: evidence from the elderly. J. Psycholinguist. Res. 24, 165-182. doi: 10.1007/BF0214 5354
Conflict of Interest Statement: The authors declare that the research was conducted in the absence of any commercial or financial relationships that could be construed as a potential conflict of interest.

The reviewer WM and handling Editor declared their shared affiliation, and the handling Editor states that the process nevertheless met the standards of a fair and objective review.

Copyright (c) 2016 Piñango, Finn, Lacadie and Constable. This is an open-access article distributed under the terms of the Creative Commons Attribution License (CC $B Y)$. The use, distribution or reproduction in other forums is permitted, provided the original author(s) or licensor are credited and that the original publication in this journal is cited, in accordance with accepted academic practice. No use, distribution or reproduction is permitted which does not comply with these terms. 